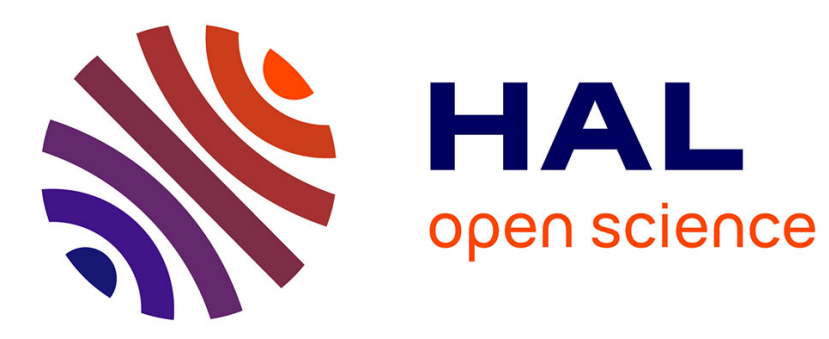

\title{
A revised Durbin-Wu-Hausman test for industrial robot identification
}

\author{
Alexandre Janot, Pierre Olivier Vandanjon, Maxime Gautier
}

\section{To cite this version:}

Alexandre Janot, Pierre Olivier Vandanjon, Maxime Gautier. A revised Durbin-Wu-Hausman test for industrial robot identification. Control Engineering Practice, 2016, 48, pp.52-62. 10.1016/j.conengprac.2015.12.017 . hal-01467446

\section{HAL Id: hal-01467446 https://hal.science/hal-01467446}

Submitted on 1 Oct 2018

HAL is a multi-disciplinary open access archive for the deposit and dissemination of scientific research documents, whether they are published or not. The documents may come from teaching and research institutions in France or abroad, or from public or private research centers.
L'archive ouverte pluridisciplinaire HAL, est destinée au dépôt et à la diffusion de documents scientifiques de niveau recherche, publiés ou non, émanant des établissements d'enseignement et de recherche français ou étrangers, des laboratoires publics ou privés. 
See discussions, stats, and author profiles for this publication at: https://www.researchgate.net/publication/289372634

\section{A revised Durbin-Wu-Hausman test for industrial robot identification}

Article in Control Engineering Practice · March 2016

Dol: 10.1016/j.conengprac.2015.12.017

CITATIONS

2

3 authors:

Alexandre Janot

The French Aerospace Lab ONERA

92 PUBLICATIONS 406 CITATIONS

SEE PROFILE

Maxime Gautier

University of Nantes

187 PUBLICATIONS 2,892 CITATIONS

SEE PROFILE

Some of the authors of this publication are also working on these related projects:

Distributed electric Propulsion for Small Business Aircraft View project

Master/Engineering Project: Sensorless Vector Control of BLDC Motors for mini-drones View project
READS

127

Pierre Olivier Vandanjon

Institut Français des Sciences et Technologies des Transports, de l'Aménagement ...

59 PUBLICATIONS 338 CITATIONS

SEE PROFILE 


\title{
A Revised Durbin-Wu-Hausman Test for Industrial Robot Identification
}

\author{
Alexandre JANOT, Pierre-Olivier VANDANJON and Maxime GAUTIER
}

Alexandre Janot (Corresponding author), French Aerospace Lab, 2 Avenue Edouard Belin, BP 74025, 31055 Toulouse Cedex 4, France; Email: Alexandre.Janot@onera.fr

Pierre Olivier Vandanjon, French Institute of Science and Technology for Transport, Development and Network (IFSTTAR), 44344 Bouguenais, France; Email: pierre-olivier.vandanjon@ifsttar.fr

Maxime Gautier, University of Nantes and IRCCyN, 1, rue de la Noë - BP 92101 - 44321 Nantes CEDEX 03, France ; Email: Maxime.Gautier@irccyn.ec-nantes.fr

\begin{abstract}
This paper addresses the topic of robot identification. The usual identification method makes use of the inverse dynamic model (IDM) and the least squares (LS) technique while robot is tracking exciting trajectories. Assuming an appropriate bandpass filtering, good results can be obtained. However, the users are in doubt whether the columns of the observation matrix (the regressors) are uncorrelated (exogenous) or correlated (endogenous) with the error terms. The exogeneity condition is rarely verified in a formal way whereas it is a fundamental condition to obtain unbiased LS estimates. In Econometrics, the Durbin-Wu-Hausman test (DWH-test) is a formal statistic for investigating whether the regressors are exogenous or endogenous. However, the DWHtest cannot be straightforwardly used for robot identification because it is assumed that the set of instruments is valid. In this paper, a Revised DWH-test suitable for robot identification is proposed. The Revised DWH-test validates/invalidates the instruments chosen by the user and validates the exogeneity assumption through the calculation of the QR factorization of the augmented observation matrix combined with a F-test if required. The experimental results obtained with a 6 degree-offreedom (DOF) industrial robot validate the proposed statistic.
\end{abstract}

Index Terms: Robots identification, Rigid robot dynamics, Instrumental variable method, Heteroskedasticity, DWH-test, Wald-statistic. 


\section{Introduction}

The usual robot identification method makes use of the continuous-time inverse dynamic model and the least squares (LS) technique while the robot is tracking some exciting trajectories. This explains why robot identification belongs to the closed-loop identification of continuous-time models from sampled data. This method, called as Inverse Dynamic Identification Model with Least Squares method (IDIM-LS), has been successfully applied to identify the inertial parameters of several prototypes and industrial robots, (Olsen et al. 2002), (Swevers et al. 2007), (Hollerbach et al. 2008), (Calanca et al. 2011), (Gautier et al. 2013) and (Janot et al. 2014, a) among others. Good results are obtained provided that an appropriate bandpass filtering of the joint positions is used to calculate the joint velocities and accelerations. However, because robots are identified in closed loop, the users can doubt whether the columns of the observation matrix (the regressors) are correlated with the error terms (endogenous) or not (exogenous) even with a data filtering, see e.g. (Söderström and Stoica 1989), (Garnier and Wang 2008), (Young 2011) and (Gilson et al. 2011).

Other identification methods were tried: the Total Least-Squares (Xi 1995), the Set Membership Uncertainty (Ramdani and Poignet 2005), an algorithm based on Linear Matrix Inequality (LMI) tools (Indri et al. 2002), a maximum likelihood (ML) approach (Olsen et al. 2002), the Closed-Loop OutputError method (Landau and Karimi 1997), (Landau 2001), (Östring et al 2003) and (Gautier et al. 2013), an algorithm based on neural network (Soewandito et al. 2011), a Bayesian approach (Ting et al. 2006), the extended Kalman filter (Gautier and Poignet 2001) and (Kostic et al. 2004), a method which estimates the nonlinear effects in the frequency domain (Wernholt and Gunnarsson 2008) and the Unscented Kalman Filter (Dellon and Matsuoka 2009). Although all these techniques are of interest, they do not really improve the IDIM-LS method combined with an appropriate data filtering. Furthermore, the robustness against data filtering was not studied, some of these approaches were not validated on a 6 degrees-of-freedom (DOF) industrial robot and the condition that the regressors are not correlated with the error terms is not addressed whereas it is a critical condition to obtain unbiased estimates (Hausman 1978), (Davidson and MacKinnon 1993) and (Wooldridge 2009). This condition is called as the exogeneity condition.

The Instrumental Variable method (IV) provides unbiased estimates while the regressors are endogenous (Söderström and Stoica 1989), (Garnier and Wang 2008) and (Young 2011). A generic IV method for industrial robots identification is proposed in (Janot et al. 2014, a) and (Janot et al. 2014, b). This approach called as the IDIM-IV method was successfully validated on a 2 DOF prototype robot and on a 6 DOF industrial robot. However, the validity of the instruments was not addressed and using the IV method while the regressors are exogenous provides inefficient unbiased estimates i.e. their variances are not minimal (Hausman 1978), (Davidson and MacKinnon 1993) and (Wooldridge 2009).

In Econometrics, the Durbin-Wu-Hausman test (DWH-test) is a formal statistic for investigating whether the regressors are exogenous or endogenous (Hausman 1978). The DWH-test makes use of the Two Stages Least Squares (2SLS) technique and an augmented LS regression. However, the DWHtest cannot be straightforwardly used for robot identification because it is implicitly assumed that the instrumental matrix is well correlated with the observation matrix and uncorrelated with the errors. Furthermore, the econometric models are empirical whereas the models used in mechanical engineering are based on physical laws (e.g. the Newton's laws). 
In this paper, it is proposed to bridge the gap between Econometrics theory and Control engineering practice by presenting a Revised DWH-test suitable for identification of robots. This revisited statistic validates/invalidates the model chosen by the user and the exogeneity condition is validated by the QR factorization of the augmented observation matrix combined with the F-test.

A condensed version of this work has been presented in (Janot et al. 2013). This paper contains detailed proofs to enlighten the theoretical understanding of the Revised DWH-test, heteroskedasticity is taken into account and additional experimental results are provided.

The rest of the paper is organized as follows. Section 2 recalls the IDIM-LS method and reviews the theory of Econometrics. Section 3 introduces the Revised DWH-test while Section 4 is devoted to experimental results. Finally, Section 5 concludes the paper.

\section{Theoretical Background: Modelling, identification of robots and Introduction of the DWH-test}

\subsection{Modelling and identification of robots}

The inverse dynamic model (IDM) of robot with $n$ moving links calculates the $(n \times 1)$ joint torques vector $\boldsymbol{\tau}_{i d m}$ as a function of generalized coordinates and their derivatives (Khalil and Dombre 2002),

$\boldsymbol{\tau}_{i d m}=\mathbf{M}(\mathbf{q}) \ddot{\mathbf{q}}+\mathbf{N}(\mathbf{q}, \dot{\mathbf{q}})$,

where $\mathbf{q}, \dot{\mathbf{q}}$ and $\ddot{\mathbf{q}}$ are respectively the $(n \times 1)$ vectors of generalized joint positions, velocities and accelerations; $\mathbf{M}(\mathbf{q})$ is the $(n \times n)$ inertia matrix; $\mathbf{N}(\mathbf{q}, \dot{\mathbf{q}})$ is the $(n \times 1)$ vector of centrifugal, Coriolis, gravitational and friction torques.

The modified Denavit and Hartenberg (MDH) notation allows to obtain an IDM which is linear in relation to a set of base parameters $\boldsymbol{\beta}$

$\boldsymbol{\tau}_{i d m}=\operatorname{IDM}(\mathbf{q}, \dot{\mathbf{q}}, \ddot{\mathbf{q}}) \boldsymbol{\beta}$,

where $\operatorname{IDM}(\mathbf{q}, \dot{\mathbf{q}}, \ddot{\mathbf{q}})$ is the $(n \times b)$ matrix of basis functions of bodies dynamics and $\boldsymbol{\beta}$ is the $(b \times 1)$ vector of base parameters.

The base parameters are the minimum number of dynamic parameters from which the IDM can be calculated. They are obtained from the standard dynamic parameters by regrouping some of them with linear relations (Mayeda et al. 1990). The standard parameters of a link $j$ are $X X_{j}, X Y_{j}, X Z_{j}$, $Y Y_{j}, Y Z_{j}$ and $Z Z_{j}$ the six components of the inertia matrix of link $j$ at the origin of frame $j ; M X_{j}$, $M Y_{j}$ and $M Z_{j}$ the components of the first moment of link $j ; M_{j}$ the mass of link $j ; I a_{j}$ a total inertia moment for rotor and gears of actuator $j ; F v_{j}$ and $F c_{j}$ the viscous and Coulomb friction parameters of joint $j$.

The direct dynamic model (DDM) of robots is given by 
$\mathbf{M}(\mathbf{q}) \ddot{\mathbf{q}}=\boldsymbol{\tau}_{i d m}-\mathbf{N}(\mathbf{q}, \dot{\mathbf{q}})$.

Proportional-Derivative (PD) and Proportional-Integral-Derivative (PID) controls are often implemented to identify the dynamic parameters. The joint $j$ signal control $v_{\tau_{j}}$ is given by

$v_{\tau_{j}}=C_{j}(s)\left(q_{r_{j}}-q_{\text {mes }_{j}}\right)$

where $C_{j}(s)$ is the transfer function of the joint $j$ controller, $q_{r_{j}}$ is the joint $j$ position reference, $q_{\text {mes }_{i}}$ is the measurement of $q_{j}$ the joint $j$ position, $s$ is the time derivative operator i.e. $s=d / d t$.

The data available from robots controllers are $\mathbf{q}_{\text {mes }}$ the $(n \times 1)$ vector of measurements of $\mathbf{q}$ and $\mathbf{v}_{\tau}$, the $(n \times 1)$ vector of control signals. The joint torques are connected with the control signals by the following relation

$\boldsymbol{\tau}=\mathbf{G}_{\tau} \mathbf{v}_{\tau}$

where $\mathbf{G}_{\tau}$ is the $(n \times n)$ diagonal matrix of drive gains. The diagonal components of $\mathbf{G}_{\tau}$ have a priori values given by manufacturers.

In (2), $\mathbf{q}$ is estimated with $\hat{\mathbf{q}}$ obtained by filtering $\mathbf{q}_{\text {mes }}$ through a lowpass Butterworth filter in both the forward and reverse directions. $(\hat{\mathbf{q}}, \hat{\mathbf{q}})$ are calculated with a central differentiation algorithm of $\hat{\mathbf{q}}$ - $\tau$ being perturbed by high-frequency disturbances, a parallel decimation procedure is used to eliminate torque ripples (see (Gautier et al. 2013) for the details).

Because of uncertainties, the $(n \times 1)$ vector of the actual joint torques $\boldsymbol{\tau}$ differs from $\boldsymbol{\tau}_{i d m}$ by an error e. The model (2) is sampled while the robot is tracking trajectories (see (Gautier et al. 2013) for the details). After data acquisition and data filtering, the following overdetermined linear system is obtained

$\mathbf{y}(\boldsymbol{\tau})=\mathbf{X}(\hat{\mathbf{q}}, \hat{\dot{\mathbf{q}}}, \hat{\hat{\mathbf{q}}}) \boldsymbol{\beta}+\boldsymbol{\varepsilon}$,

where $\mathbf{y}(\boldsymbol{\tau})$ is the $(r \times 1)$ measurements vector built from the actual torques $\boldsymbol{\tau} ; \mathbf{X}(\hat{\mathbf{q}}, \hat{\mathbf{\mathbf { q }}}, \hat{\hat{\mathbf{q}}})$ is the $(r \times b)$ observation matrix built from the sampling of $\operatorname{IDM}(\hat{\mathbf{q}}, \hat{\mathbf{q}}, \hat{\mathbf{q}}) ; \boldsymbol{\varepsilon}$ is the $(r \times 1)$ sampled vector of e $r=n \cdot n_{e}$ is the number of rows in (6), $n_{e}$ being the number of rows in a subsystem $j$.

Relation (6) is the Inverse Dynamic Identification Model (IDIM). The columns of $\mathbf{X}(\hat{\mathbf{q}}, \hat{\dot{\mathbf{q}}}, \hat{\mathbf{q}})$ are the regressors. $\boldsymbol{\varepsilon}$ is assumed to have zero mean, to be serially uncorrelated with a covariance matrix $\boldsymbol{\Omega}$

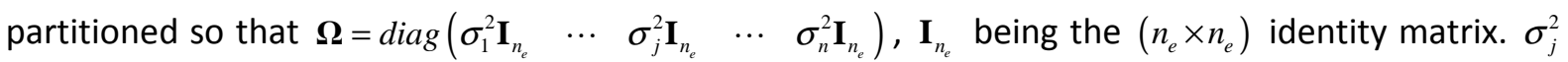
is estimated through the Ordinary Least Squares (OLS) solution of a subsystem $j$ (see (Gautier et al. 2013) for the details). The IDIM-LS estimates and their covariance matrix are given by

$\hat{\boldsymbol{\beta}}_{L S}=\left(\mathbf{X}^{T} \mathbf{\Omega}^{-1} \mathbf{X}\right)^{-1} \mathbf{X}^{T} \mathbf{\Omega}^{-1} \mathbf{y}, \hat{\boldsymbol{\Sigma}}_{L S}=\left(\mathbf{X}^{T} \mathbf{\Omega}^{-1} \mathbf{X}\right)^{-1}$. 
The IDIM-LS estimates are unbiased if

$E\left(\mathbf{X}^{T} \boldsymbol{\varepsilon}\right)=\mathbf{0}$,

where $E($.$) is the expectation operator (Davidson and MacKinnon 1993).$

Because robots are identified in closed loop, the users can doubt whether $\mathbf{X}(\hat{\mathbf{q}}, \hat{\mathbf{q}}, \hat{\mathbf{q}})$ is correlated with $\varepsilon$ or not. To overcome the problem of a correlation between $\mathbf{X}$ and $\varepsilon$, the Two-Stage-LeastSquares (2SLS) technique is an appropriate method.

\subsection{Review of theory of Econometrics}

The 2SLS method estimates $\boldsymbol{\beta}$ with two LS regressions. Researchers in Econometrics consider the model (6) as the reduced form of the general model defined by

$\left\{\begin{array}{l}\mathbf{y}=\mathbf{X} \boldsymbol{\beta}+\boldsymbol{\varepsilon} \\ \mathbf{X}=\mathbf{Z} \boldsymbol{\Pi}+\mathbf{V}^{\prime}\end{array}\right.$

where $\mathbf{Z}$ is the $(r \times z)$ instrumental matrix with $z \geq b ; \boldsymbol{\Pi}$ is the $(z \times b)$ matrix of coefficients to be identified and $\mathbf{V}$ is a $(r \times b)$ matrix of error terms.

The columns of $\mathbf{Z}$ are called instruments. If the following assumptions hold $\operatorname{rank}(\mathbf{Z})=b, E\left(\mathbf{Z}^{T} \boldsymbol{\varepsilon}\right)=\mathbf{0}$ ,$E\left(\mathbf{Z}^{T} \mathbf{V}\right)=\mathbf{0}$ and $E(\mathbf{V})=\mathbf{0}, \mathbf{Z}$ is said valid.

The first stage calculates $\hat{\boldsymbol{\Pi}}$, the LS estimate of $\boldsymbol{\Pi}$, given by $\hat{\mathbf{\Pi}}=\left(\mathbf{Z}^{T} \mathbf{Z}\right)^{-1} \mathbf{Z}^{T} \mathbf{X}$. $\hat{\mathbf{X}}$, the projected of $\mathbf{X}$ onto the space spanned by the columns of $\mathbf{Z}$, is given by

$\hat{\mathbf{X}}=\mathbf{Z} \hat{\mathbf{\Pi}}=\mathbf{Z}\left(\mathbf{Z}^{T} \mathbf{Z}\right)^{-1} \mathbf{Z}^{T} \mathbf{X}=\mathbf{P}_{\mathbf{Z}} \mathbf{X}$

where $\mathbf{P}_{Z}=\mathbf{Z}\left(\mathbf{Z}^{T} \mathbf{Z}\right)^{-1} \mathbf{Z}^{T}$ is the idempotent $(r \times r)$ projection matrix of $\mathbf{Z}$.

The second stage calculates the 2SLS estimates. Assuming that $\mathbf{X}^{T} \mathbf{P}_{Z} \mathbf{X}=\hat{\mathbf{X}}^{T} \hat{\mathbf{X}}$ is nonsingular i.e. $\operatorname{rank}(\hat{\mathbf{X}})=b$, the 2 SLS estimates and their associated covariance matrix are given by (Wooldridge 2009)

$\hat{\boldsymbol{\beta}}_{2 S L S}=\left(\hat{\mathbf{X}}^{T} \boldsymbol{\Omega}^{-1} \hat{\mathbf{X}}\right)^{-1} \hat{\mathbf{X}}^{T} \boldsymbol{\Omega}^{-1} \mathbf{y}, \hat{\boldsymbol{\Sigma}}_{2 S L S}=\left(\hat{\mathbf{X}}^{T} \boldsymbol{\Omega}^{-1} \hat{\mathbf{X}}\right)^{-1}$

If $z=b$ the 2SLS estimates collapse to the IV estimates given by $\hat{\boldsymbol{\beta}}_{I V}=\left(\mathbf{Z}^{T} \mathbf{X}\right)^{-1} \mathbf{Z}^{T} \mathbf{y}$.

If the 2SLS method is used while relation (8) holds, the estimates are unbiased but their variances are not minimal (Hausman 1978), (Davidson and MacKinnon 1993) and (Wooldridge 2009). The DurbinWu-Hausman test (DWH-test) is a formal test which examines whether (8) holds or not. This paper focuses on the augmented DWH-test (Hausman 1978). Assuming that $\mathbf{Z}$ is valid, the model (9) can 
be written as $\mathbf{y}=\hat{\mathbf{X}} \boldsymbol{\beta}+\mathbf{V} \boldsymbol{\beta}+\boldsymbol{\varepsilon}$. Then, by referring to the coefficient corresponding to $\mathbf{V}$ as $\gamma$ and rewriting (9) after adding and subtracting $\mathbf{V} \boldsymbol{\beta}$, one obtains $\mathbf{y}=(\hat{\mathbf{X}}+\mathbf{V}) \boldsymbol{\beta}+\mathbf{V}(\boldsymbol{\gamma}-\boldsymbol{\beta})+\boldsymbol{\varepsilon}=\mathbf{X} \boldsymbol{\beta}+\mathbf{V} \boldsymbol{\theta}+\boldsymbol{\varepsilon}$, with $\boldsymbol{\theta}=\boldsymbol{\gamma}-\boldsymbol{\beta}$ being the $(b \times 1)$ vector of omitted parameters that explain the correlation between $\mathbf{X}$ and $\boldsymbol{\varepsilon}$. The following relation called as "exogeneity condition" is obtained

$$
E\left(\mathbf{X}^{T} \boldsymbol{\varepsilon}\right)=\mathbf{0} \Leftrightarrow \hat{\boldsymbol{\theta}}=\mathbf{0}
$$

Because $\mathbf{V}$ is not known, its estimate is calculated with $\hat{\mathbf{V}}=\mathbf{X}-\mathbf{Z} \hat{\mathbf{\Pi}}$ and the following augmented regression is built $\mathbf{y}=\left[\begin{array}{ll}\mathbf{X} & \hat{\mathbf{V}}\end{array}\right]\left[\begin{array}{l}\boldsymbol{\beta} \\ \boldsymbol{\theta}\end{array}\right]+\boldsymbol{\varepsilon}$. The LS estimates $\hat{\boldsymbol{\beta}}$ and $\hat{\boldsymbol{\theta}}$ are then calculated and with an appropriate statistical test (e.g. F-test), it is checked that the null hypothesis $H_{0}: \hat{\boldsymbol{\theta}}=\mathbf{0}$ holds. If the test accepts $H_{0}$, the LS estimates are unbiased, otherwise they are biased (Hausman 1978) and (Wooldridge 2009).

Although the DWH-test is of great interest, it cannot be used as it is. First, the unbiasedness of the 2SLS estimates and the DWH-test are based on the fact that the $\mathbf{Z}$ is valid. In practice, how to validate/invalidate this assumption? Second, the DWH-test can detect a bias of the LS estimator but it cannot provide the origin of this bias. Third, the models used in Econometrics are empirical whereas the models used in Mechanical/Electrical Engineering are mostly based on physical laws. Fourth, the notion of closed-loop identification is not addressed in Econometrics. In the following section, a Revised DWH-test that validates/invalidates the construction of $\mathbf{Z}$ and determinates the origin of the bias of LS estimates is presented.

\section{A Statistic to Validate/Invalidate the IDIM-LS Estimates}

\subsection{Preliminary definitions}

Because of noisy measurements, the following definitions are introduced $q_{\text {mes }_{j}}=q_{n f_{j}}+\delta q_{\text {mes }_{j}}$, $\tau_{j}=\tau_{n f_{j}}+\delta \tau_{j}+\delta \tau_{q_{j}}, \hat{q}_{j}=q_{n f_{j}}+\delta \hat{q}_{j}, \hat{\dot{q}}_{j}=\dot{q}_{n f_{j}}+\delta \hat{\dot{q}}_{j}$ and $\hat{\ddot{q}}_{j}=\ddot{q}_{n f_{j}}+\delta \hat{\ddot{q}}_{j}, q_{n f_{j}}, \dot{q}_{n f_{j}}, \ddot{q}_{n f_{j}}$ are the joint $j$ noisefree position, velocity and acceleration respectively, $\tau_{n f_{j}}$ is the joint $j$ noise-free torque given by $\tau_{n f_{j}}=g_{\tau_{j}} C(s)\left(q_{r_{j}}-q_{n f_{j}}\right), \delta q_{\text {mes }_{j}}$ is the measurement error, $\delta \hat{q}_{j}, \delta \hat{\dot{q}}_{j}$ and $\delta \hat{\ddot{q}}_{j}$ are the errors in $\hat{q}_{j}, \hat{\dot{q}}_{j}$ and $\hat{\ddot{q}}_{j}$ respectively. At last $\delta \tau_{q_{j}}=g_{\tau_{j}} C(s) \delta q_{\text {mes }_{j}}$ is the error in $\tau_{j}$ due to the feedback and $\delta \tau_{j}$ is the error in $\tau_{j}$ due to the measurement noise.

Let $\mathbf{e}_{\tau}=\left[\begin{array}{lll}\delta \tau_{1} & \cdots & \delta \tau_{n}\end{array}\right]^{T}$ be the $(n \times 1)$ vector of measurements noises in $\boldsymbol{\tau}, \mathbf{e}_{q_{m e s}}=\left[\begin{array}{lll}\delta \tau_{q_{1}} & \cdots & \delta \tau_{q_{n}}\end{array}\right]^{T}$ be the $(n \times 1)$ vector of measurements noises in $\boldsymbol{\tau}$ due to $\delta \mathbf{q}_{\text {mes }}=\left[\begin{array}{lll}\delta q_{\text {mes }_{1}} & \cdots & \delta q_{\text {mes }_{n}}\end{array}\right]^{T}$ the $(n \times 1)$ vector of measurements noises in $\mathbf{q}_{\text {mes }}$. Let $\delta \hat{\mathbf{q}}, \delta \hat{\dot{\mathbf{q}}}$ and $\delta \hat{\hat{\mathbf{q}}}$ be the $(n \times 1)$ vector of noises in $\hat{\mathbf{q}}, \hat{\mathbf{q}}$ and $\hat{\hat{\mathbf{q}}}$ respectively with $\delta \hat{\mathbf{q}}=\left[\begin{array}{lll}\delta \hat{q}_{1} & \cdots & \delta \hat{q}_{n}\end{array}\right]^{T}, \delta \hat{\hat{\mathbf{q}}}=\left[\begin{array}{lll}\delta \hat{q}_{1} & \cdots & \delta \hat{\dot{q}}_{n}\end{array}\right]^{T}$ and $\delta \hat{\hat{\mathbf{q}}}=\left[\begin{array}{lll}\delta \hat{\ddot{q}}_{1} & \cdots & \delta \hat{\ddot{q}}_{n}\end{array}\right]^{T}$. Let $\mathbf{q}_{n f}, \dot{\mathbf{q}}_{n f}, \ddot{\mathbf{q}}_{n f}$ be the $(n \times 1)$ vector of noise-free positions, velocities and accelerations respectively. 
Since $\hat{\mathbf{q}}$ is obtained through the filtering of $\mathbf{q}_{\text {mes }}$ and since $(\hat{\dot{\mathbf{q}}}, \hat{\mathbf{q}})$ are calculated from the differentiation of $\hat{\mathbf{q}}$, the errors $\delta \mathbf{q}_{\text {mes }}$ and $\delta \hat{\mathbf{q}}, \delta \hat{\dot{\mathbf{q}}}, \delta \hat{\hat{\mathbf{q}}}$ are correlated.

\subsection{Exogeneity condition for robot identification}

For robot identification, the true model is assumed to be

$$
\left\{\begin{array}{l}
\mathbf{y}=\mathbf{X}_{n f} \boldsymbol{\beta}+\boldsymbol{\varepsilon}_{q}+\boldsymbol{\varepsilon}_{\tau}, \\
\mathbf{X}=\mathbf{X}_{n f}+\mathbf{V}
\end{array},\right.
$$

where $\mathbf{X}_{n f}$ is the $(r \times b)$ noise-free observation matrix built from the sampling of $\operatorname{IDM}\left(\mathbf{q}_{n f}, \dot{\mathbf{q}}_{n f}, \ddot{\mathbf{q}}_{n f}\right)$, $\boldsymbol{\varepsilon}_{\tau}$ is the $(r \times 1)$ sampled vector of $\mathbf{e}_{\tau} ; \boldsymbol{\varepsilon}_{q}$ is the $(r \times 1)$ sampled vector of $\mathbf{e}_{q_{m s}} ; \mathbf{V}$ is the $(r \times b)$ matrix of error terms that depends on the sampling of $\delta \hat{\mathbf{q}}, \delta \hat{\mathbf{q}}, \delta \hat{\hat{\mathbf{q}}}$.

With $E\left(\boldsymbol{\varepsilon}_{q}\right)=E\left(\boldsymbol{\varepsilon}_{\tau}\right)=\mathbf{0}, E(\mathbf{V})=\mathbf{0}$ and $\boldsymbol{\varepsilon}_{\tau}$ being uncorrelated with $\boldsymbol{\varepsilon}_{q}$, one obtains $E\left(\mathbf{V}^{T} \boldsymbol{\varepsilon}_{\tau}\right)=$ $E\left(\mathbf{V}^{T}\right) E\left(\boldsymbol{\varepsilon}_{\tau}\right)=\mathbf{0}$ and $E\left(\boldsymbol{\varepsilon}_{q}^{T} \boldsymbol{\varepsilon}_{\tau}\right)=E\left(\boldsymbol{\varepsilon}_{q}^{T}\right) E\left(\boldsymbol{\varepsilon}_{\tau}\right)=0$. Because $\delta \mathbf{q}_{\text {mes }}$ and $\delta \hat{\mathbf{q}}, \delta \hat{\mathbf{q}}, \delta \hat{\mathbf{q}}$ are correlated, $\boldsymbol{\varepsilon}_{q}$ and $\mathbf{V}$ are also correlated. As usually done in Statistics, we introduce $\boldsymbol{\varepsilon}_{q}=\mathbf{V} \boldsymbol{\gamma}^{\prime}$ where $\boldsymbol{\gamma}^{\prime}$ is the $(b \times 1)$ vector of parameters that explain the correlation between $\mathbf{V}$ and $\boldsymbol{\varepsilon}_{q}$. With $\mathbf{X}_{n f}=\mathbf{X}-\mathbf{V}$ and by introducing $\boldsymbol{\theta}=\boldsymbol{\gamma}^{\prime}-\boldsymbol{\beta}$ the $(b \times 1)$ vector of omitted variables, it yields $\boldsymbol{\varepsilon}=\boldsymbol{\varepsilon}_{\tau}+\mathbf{V} \boldsymbol{\theta}$. After calculations, one obtains $E\left(\mathbf{X}^{T} \boldsymbol{\varepsilon}\right)=E\left(\mathbf{V}^{T} \mathbf{V}\right) \boldsymbol{\theta}$.

$E\left(\mathbf{X}^{T} \boldsymbol{\varepsilon}\right)=\mathbf{0}$ implies two exogeneity conditions

$\boldsymbol{\theta}=\mathbf{0}$,

or

$\mathbf{V}=\mathbf{0}$.

$\gamma^{\prime}$ being the vector of parameters that have no real physical meaning, $\gamma^{\prime}$ and $\beta$ are not of the same nature in the case of robot identification and relation (14) is quite implausible. Furthermore, by calculating $\hat{\mathbf{q}}$ through the filtering of $\mathbf{q}_{\text {mes }}$ and by calculating $(\hat{\dot{\mathbf{q}}}, \hat{\mathbf{q}})$ from the differentiation of $\hat{\mathbf{q}}$, the relations $\delta \hat{\mathbf{q}} \approx \mathbf{0}, \delta \hat{\dot{\mathbf{q}}} \approx \mathbf{0}, \delta \hat{\ddot{\mathbf{q}}} \approx \mathbf{0}$ are expected. $\mathbf{V}$ being built from the sampling of $\delta \hat{\mathbf{q}}, \delta \hat{\dot{\mathbf{q}}}, \delta \hat{\ddot{\mathbf{q}}}$, relation (15) is the expected relation.

Another way of looking at (15) is the design of the right inputs (also called 'optimal trajectories' in robotics) that allow to obtain the best estimates. This is the experiment design (Aguero and Goodwin 2006) and (Aguero and Goodwin 2007). The works presented in these references cannot be straightforwardly applied for robot identification because robots are nonlinear Multi-Input-MultiOutput (MIMO) systems whereas the works presented in these references are focussed on linear Single-Input-Single-Output (SISO) systems. At last, the basis functions contain nonlinear functions. Those reasons explain why the authors suggest to run the proposed approach. 
According to (Gautier 1991), (15) is equivalent to state that $\boldsymbol{\theta}$ has no influence on robot dynamics. To assess the influence of $\boldsymbol{\theta}$, (6) is first rewritten as $\mathbf{y}=\left[\begin{array}{ll}\mathbf{X} & \mathbf{V}\end{array}\right]\left[\begin{array}{l}\boldsymbol{\beta} \\ \boldsymbol{\theta}\end{array}\right]+\boldsymbol{\varepsilon}_{\tau}=\mathbf{X}_{X T D} \boldsymbol{\beta}_{X T D}+\boldsymbol{\varepsilon}_{\tau}$ where $\mathbf{X}_{X T D}=\left[\begin{array}{ll}\mathbf{X} & \mathbf{V}\end{array}\right]$ is the $(r \times 2 b)$ augmented observation matrix and $\boldsymbol{\beta}_{X T D}=\left[\begin{array}{ll}\boldsymbol{\beta}^{T} & \boldsymbol{\theta}^{T}\end{array}\right]^{T}$ is the $(2 b \times 1)$ augmented vector of parameters. Second, the QR decomposition of $\mathbf{X}_{X T D}$ is considered. This gives

$\mathbf{X}_{X T D}=\mathbf{Q}_{\mathbf{X}_{X T D}}\left[\begin{array}{c}\mathbf{R}_{\mathbf{X}_{X T D}} \\ \mathbf{0}_{(r-2 b) \times 2 b}\end{array}\right]$,

where $\mathbf{Q}_{\mathbf{X}_{X T D}}$ is a $(r \times r)$ orthogonal matrix i.e. $\mathbf{Q}_{\mathbf{X}_{X T D}^{T}}^{T} \mathbf{Q}_{\mathbf{X}_{X T D}}=\mathbf{I}_{r}$, and $\mathbf{R}_{\mathbf{X}_{X T D}}$ is a $(2 b \times 2 b)$ upper triangular matrix.

Third, let $r_{\mathrm{x}}^{k}$ (resp. $r_{\mathrm{v}}^{k}$ ) be the absolute value of the $b$ first (resp. last) diagonal elements of $\mathbf{R}_{\mathbf{x}_{x T D}}$ i.e. $r_{\mathbf{X}}^{k}=\left|R_{\mathbf{X}_{X T D}}(k, k)\right|$ for $k=1, \ldots, b$ (resp. $r_{\mathrm{v}}^{k}=\left|R_{\mathbf{X}_{X T D}}(k, k)\right|$ for $\left.k=b+1, \ldots, 2 b\right)$. According to (Gautier 1991), $\boldsymbol{\theta}$ has no influence if all $r_{\mathrm{v}}^{k}$ 's are null

$r_{\mathrm{v}}^{k}=0$ for $k=1, \ldots, b$.

In this case, (15) holds because $\mathbf{X}_{X T D}$ is rank deficient and collapses to $\mathbf{X}$.

Fourth, if all or some $r_{\mathrm{v}}^{k}$ 's are not null, then $\boldsymbol{\theta}$ may significantly contribute to robot dynamics. To assess this contribution and to make a final decision, a F-test associated with the following hypothesis $H_{0}: \boldsymbol{\theta}=\mathbf{0}$ is run. If the F-test accepts $H_{0}$, then the LS estimates are unbiased; otherwise they are biased.

In this section, the exogeneity condition for robot identification has been given. However, it is assumed that a valid instrumental matrix $\mathbf{Z}$ exists. In the following section, it is explained how to construct $\mathbf{Z}$ and how to validate/invalidate this construction.

\subsection{Construction and validation/invalidation of an instrumental matrix}

In (Janot et al. 2014, a), it has been shown that a $(r \times b)$ valid instrumental matrix is

$\mathbf{Z}=\mathbf{X}_{n f}=\mathbf{X}\left(\mathbf{q}_{n f}, \dot{\mathbf{q}}_{n f}, \ddot{\mathbf{q}}_{n f}\right)$.

where $\mathbf{X}\left(\mathbf{q}_{n f}, \dot{\mathbf{q}}_{n f}, \ddot{\mathbf{q}}_{n f}\right)$ is the $(r \times b)$ sampled matrix of $\operatorname{IDM}\left(\mathbf{q}_{n f}, \dot{\mathbf{q}}_{n f}, \ddot{\mathbf{q}}_{n f}\right)$.

To build $\mathbf{Z}$, the DDM given by (3) is simulated with the previous IV estimates denoted as $\hat{\boldsymbol{\beta}}_{I V}^{i t-1}$ and assuming the same references and the same control law structure for both the actual and the simulated robots. $\ddot{\mathbf{q}}_{s}$ the vector of the simulated joint accelerations is given by $\mathbf{M}\left(\mathbf{q}_{S}, \hat{\boldsymbol{\beta}}_{I V}^{i t-1}\right) \ddot{\mathbf{q}}_{S}=\boldsymbol{\tau}_{S}-\mathbf{N}\left(\mathbf{q}_{S}, \dot{\mathbf{q}}_{S}, \hat{\boldsymbol{\beta}}_{I V}^{i t-1}\right)$ where $\mathbf{q}_{S}, \dot{\mathbf{q}}_{S}$ are respectively the $(n \times 1)$ vectors of the simulated joint positions and velocities calculated by numerical integration of the DDM while $\boldsymbol{\tau}_{s}$ is the $(n \times 1)$ vector of simulated torques with $\tau_{S_{j}}$, the $\mathrm{j}^{\text {th }}$ element of $\boldsymbol{\tau}_{S}$, is given by $\tau_{s_{j}}=g_{\tau_{j}} C_{j}(s)\left(q_{r_{j}}-q_{S_{j}}\right)$. 
Let $\hat{\mathbf{Z}}$ defined by

$\hat{\mathbf{Z}}=\mathbf{X}\left(\mathbf{q}_{S}, \dot{\mathbf{q}}_{s}, \ddot{\mathbf{q}}_{S}, \hat{\boldsymbol{\beta}}_{I V}^{i t-1}\right)$

where $\mathbf{X}\left(\mathbf{q}_{S}, \dot{\mathbf{q}}_{S}, \ddot{\mathbf{q}}_{S}, \hat{\boldsymbol{\beta}}_{I V}^{i t-1}\right)$ is the $(r \times b)$ sampled matrix of $\operatorname{IDM}\left(\mathbf{q}_{S}, \dot{\mathbf{q}}_{S}, \ddot{\mathbf{q}}_{S}, \hat{\boldsymbol{\beta}}_{I V}^{i t-1}\right)$.

At iteration $i t$, the IV estimates are given by

$\hat{\boldsymbol{\beta}}_{I V}^{i t}=\left(\hat{\mathbf{Z}}^{T} \mathbf{X}\right)^{-1} \hat{\mathbf{Z}}^{T} \mathbf{y}$

In order to ensure $\hat{\mathbf{Z}} \approx \mathbf{X}\left(\mathbf{q}_{n f}, \dot{\mathbf{q}}_{n f}, \ddot{\mathbf{q}}_{n f}\right) \forall \hat{\boldsymbol{\beta}}_{I V}^{i t-1}$, the gains of the simulated controller of the simulated robot are updated according to $\hat{\boldsymbol{\beta}}_{V V}^{i t}$. The updating procedure is completely described in (Janot et al. 2014, a) and (Janot et al. 2014, b). According to the results presented in (Janot et al. 2014, a), this IV approach can be considered as a one-step IV algorithm. Consequently, a one-step 2SLS algorithm is considered for experiments.

It is now shown how to validate/invalidate the construction of $\hat{\mathbf{Z}}$. With $\mathbf{Z}=\mathbf{X}_{n f}$, the following equality holds $\boldsymbol{\Pi}=\mathbf{I}_{b}$ where $\mathbf{I}_{b}$ is the $(b \times b)$ identity matrix. $\hat{\boldsymbol{\Pi}}_{\text {exp }}$ the expected value of $\hat{\boldsymbol{\Pi}}$ the estimate of $\boldsymbol{\Pi}$ is defined by $\hat{\boldsymbol{\Pi}}_{\exp }=\mathbf{I}_{b} . \hat{\boldsymbol{\pi}}_{k-\exp }$ the expected value of the $\mathbf{k}^{\text {th }}$ column of $\hat{\boldsymbol{\Pi}}$ is defined as $\hat{\boldsymbol{\pi}}_{k-\exp }(i)=1$ for $i=k$ and $\hat{\boldsymbol{\pi}}_{k-\exp }(i)=0$ for $i \neq k$.

$\hat{\boldsymbol{\pi}}_{k}$ the $\mathrm{k}^{\text {th }}$ column of $\hat{\boldsymbol{\Pi}}$ is calculated with $\hat{\boldsymbol{\pi}}_{k}=\left(\hat{\mathbf{Z}}^{T} \hat{\mathbf{Z}}\right)^{-1} \hat{\mathbf{Z}}^{T} \mathbf{x}_{k}$ where $\mathbf{x}_{k}$ is the $\mathrm{k}^{\text {th }}$ column of $\mathbf{X} . \hat{\mathbf{v}}_{k}$ the $\mathbf{k}^{\text {th }}$ column of $\hat{\mathbf{v}}$ is given by $\hat{\mathbf{v}}_{k}=\hat{\mathbf{Z}} \hat{\boldsymbol{\pi}}_{k}-\mathbf{x}_{k}$. It is assumed that $\hat{\mathbf{v}}_{k} \sim N\left(\mathbf{0}, \boldsymbol{\Omega}_{\hat{\mathbf{v}}_{k}}\right)$ where $\boldsymbol{\Omega}_{\hat{\mathbf{v}}_{k}}$ is a diagonal matrix whose the diagonal elements are unknown to the users. In (White 1980), the author showed that the $\mathrm{i}^{\text {th }}$ diagonal element of $\boldsymbol{\Omega}_{\hat{\mathbf{v}}_{k}}$ can be estimated with $\hat{\boldsymbol{\Omega}}_{\hat{\mathbf{v}}_{k}}(i, i)=\hat{\mathbf{v}}_{k}^{2}(i), \hat{\mathbf{v}}_{k}(i)$ being the $\mathbf{i}^{\text {th }}$ element of $\hat{\mathbf{v}}_{k}$. The estimated covariance matrix of $\hat{\boldsymbol{\pi}}_{k}^{j}$ is then given by

$\hat{\mathbf{\Sigma}}_{\hat{\pi}_{k} \hat{\pi}_{k}}=\left(\hat{\mathbf{Z}}^{T} \hat{\mathbf{Z}}\right)^{-1} \hat{\mathbf{Z}}^{T} \hat{\mathbf{\Omega}}_{\hat{\boldsymbol{v}}_{k}} \hat{\mathbf{Z}}\left(\hat{\mathbf{Z}}^{T} \hat{\mathbf{Z}}\right)^{-1}$

Then, the following Wald-statistic is calculated

$\eta_{\hat{\mathbf{\delta}}}^{2}=\hat{\boldsymbol{\delta}}_{\pi_{k}}^{T} \hat{\boldsymbol{\Sigma}}_{\hat{\pi}_{k} \hat{\pi}_{k}}^{-1} \hat{\boldsymbol{\delta}}_{\pi_{k}}$

where $\hat{\boldsymbol{\delta}}_{\boldsymbol{\pi}_{k}}=\hat{\boldsymbol{\pi}}_{k}-\hat{\boldsymbol{\pi}}_{k-\exp }$.

If $\eta_{\hat{\mathbf{\delta}}}^{2} \leq \chi^{2}(b)$ for a level of significance $\alpha$ usually chosen between 0.1 and $0.01, H_{0}: \hat{\pi}_{k}=\hat{\pi}_{k-\exp }$ holds. The construction is $\hat{\mathbf{Z}}$ validated. Otherwise, this construction is invalidated.

Relation (23) indicates if the distance between $\hat{\boldsymbol{\pi}}_{k}$ and $\hat{\boldsymbol{\pi}}_{k-\exp }$ is compatible the variances calculated. If the Wald-test accepts $H_{0}: \hat{\boldsymbol{\pi}}_{k}=\hat{\boldsymbol{\pi}}_{k-\exp }$ for all $k$, then the relation $\hat{\boldsymbol{\Pi}}=\hat{\boldsymbol{\Pi}}_{\text {exp }}$ is verified and that proves that the statistical assumption made on $\hat{\mathbf{V}}$ hold. Indeed, if (23) holds, $\hat{\boldsymbol{\pi}}_{k}$ is a consistent 
estimate of $\hat{\boldsymbol{\pi}}_{k-\exp }$ and there exists a compact neighbourhood such that $\left|\hat{\boldsymbol{\pi}}_{k}-\hat{\boldsymbol{\pi}}_{k-\exp }\right|$ is finite. Because the trajectories are bounded and according to the results exposed in (White 1980), it follows that $\hat{\mathbf{v}}_{k}$ is a consistent estimate of $\mathbf{v}_{k}$. Since $E(\mathbf{V})=\mathbf{0}$ implies $E\left(\mathbf{v}_{k}\right)=\mathbf{0}$, one obtains $E\left(\hat{\mathbf{v}}_{k}\right)=\mathbf{0}$ for all $k$ and this leads to $E(\hat{\mathbf{V}})=\mathbf{0}$.

\subsection{Algorithm of the Revised DWH-test for robot identification}

The Revised DWH-test is run as follows (see Fig. 1):

1. Construct the instrumental variable matrix $\hat{\mathbf{Z}}$ and validate/invalidate this construction with the algorithm described in Section 3.3.

2. If $\hat{\mathbf{Z}}$ is valid, calculate $\hat{\mathbf{V}}=\mathbf{X}-\hat{\mathbf{Z}}$.

3. Check with the QR decomposition of $\mathbf{X}_{X T D}$ that $\boldsymbol{\theta}$ has no influence on robot dynamics as explained in Section 3.2.

4. If the $r_{\mathrm{v}}^{k}$ 's are not null, assess the contribution of $\boldsymbol{\theta}$ thanks to a F-test associated with $H_{0}: \boldsymbol{\theta}=\mathbf{0}$. If the F-test accepts $H_{0}$, then the LS estimates are considered as unbiased; otherwise, they are biased.

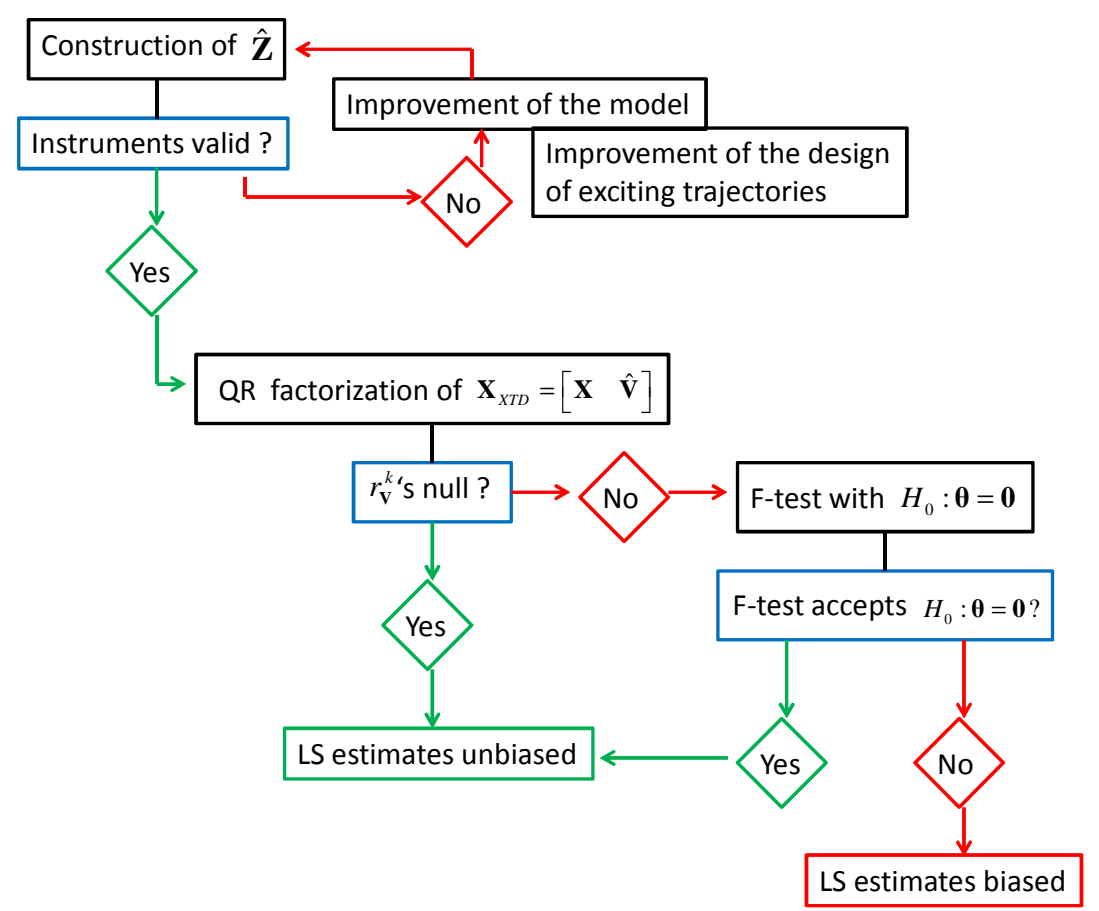

Fig. 1. Scheme of the Revised DWH-test suitable for robot identification

Compared with the classical regressed DWH-test, the Revised DWH-test can determine the origin of 
the bias by evaluating the validity of the instruments, can detect a model misspecification and combines the QR factorization with a F-test. Those remarks make the proposed statistic relevant for mechatronic system identification.

\section{Experimental Identification Results Obtained with the TX40}

\subsection{Model Reduction and Validation of the Statistical Hypotheses}

Before presenting the experimental results obtained with the TX40 robot, the F-test used to eliminate the dynamic parameters having no effect on robot dynamics is first introduced. Then, the tests which validate/invalidate the statistical assumptions are presented.

\subsubsection{F-test}

Some dynamic parameters remain poorly identifiable because they are small. They can be cancelled to simplify the inverse and direct models. The most rigorous way consists in using the F-test (Davidson and MacKinnon 1993) which is carried out with the weighted error $\overline{\boldsymbol{\varepsilon}}=\mathbf{\Omega}^{-1 / 2} \boldsymbol{\varepsilon}$. Because $E\left(\overline{\boldsymbol{\varepsilon}}^{T}\right)=\boldsymbol{\Omega}^{-1 / 2} E\left(\boldsymbol{\varepsilon} \boldsymbol{\varepsilon}^{T}\right) \boldsymbol{\Omega}^{-1 / 2}=\boldsymbol{\Omega}^{-1 / 2} \boldsymbol{\Omega} \boldsymbol{\Omega}^{-1 / 2}=\mathbf{I}_{r}$, it is assumed that $\overline{\boldsymbol{\varepsilon}} \sim \mathrm{N}\left(\mathbf{0}, \mathbf{I}_{r}\right)$ and the samples of $\overline{\boldsymbol{\varepsilon}}$ are independent. From $b$ base parameters, $b c$ parameters may define the set of essential parameters that is enough to describe the robot dynamics. The F-test is performed as follows:

1. First, one runs the 2 SLS method with the $b$ base parameters and one computes $\|\bar{\varepsilon}\|$;

2. Second, one runs the 2SLS method with the $b c$ essential parameters and one computes $\left\|\overline{\boldsymbol{\varepsilon}}_{c}\right\|$, the error norm obtained with the reduced model;

3. Third, one calculates

$\hat{F}=\frac{\left(\left\|\overline{\boldsymbol{\varepsilon}_{c}}\right\|^{2}-\|\overline{\boldsymbol{\varepsilon}}\|^{2}\right) /(b-b c)}{\|\overline{\boldsymbol{\varepsilon}}\|^{2} /(r-b)}$.

If $\hat{F}$ is less than $F_{(1-\alpha),(b-b c),(r-b)}$, the F-test accepts the model reduction; otherwise, it is rejected.

The F-test works if $\overline{\boldsymbol{\varepsilon}} \sim \mathrm{N}\left(\mathbf{0}, \mathbf{I}_{r}\right)$ holds and if the samples of $\overline{\boldsymbol{\varepsilon}}$ are independent. These assumptions must be validated with the Kolmogorov-Smirnov test (KS-test) and the Durbin-Watson test (DW-test).

\subsubsection{Kolmogorov-Smirnov test (KS-test)}

The KS-test is a nonparametric test for equality of continuous one dimensional probability distribution that can be used to compare a sample with a reference probability distribution. The KStest quantifies a distance between the empirical distribution function (EDF) of the sample and the cumulative distribution function (CDF) of the reference distribution. In our case, the null hypothesis is $H_{0}: \overline{\boldsymbol{\varepsilon}} \sim \mathrm{N}\left(\mathbf{0}, \mathbf{I}_{r}\right)$. The EDF of $\overline{\boldsymbol{\varepsilon}}$ is compared with the CDF of the reference distribution via a KS-test with a 0.05 level of significance. 


\subsubsection{DW-test}

Assuming $\overline{\boldsymbol{\varepsilon}} \sim \mathrm{N}\left(\mathbf{0}, \mathbf{I}_{r}\right)$, the DW-statistic is given by

$d w=\sum_{i=2}^{r}(\bar{\varepsilon}(i)-\bar{\varepsilon}(i-1))^{2} / \sum_{i=1}^{r} \bar{\varepsilon}(i)^{2} \approx 2\left(1-\rho_{1}\right)$

where $\rho_{1}$ is the sample autocorrelation and $\bar{\varepsilon}(i)$ is the $\mathrm{i}^{\text {th }}$ sample of $\bar{\varepsilon}$.

The value of $d w$ lies between 0 and 4. $d w=2$ indicates no autocorrelation i.e. $\rho_{1}=0$ and if the DWstatistic is substantially less than 2 , there is evidence of positive serial correlation. Small values of $d w$ indicate that successive error terms are close in value to one another (or positively correlated). Similarly, if $d w$ is greater than 2, successive error terms are much different in value from one another (negatively correlated).

For robot identification, as a rough rule of thumb, if $d w$ varies between 1.8 and $2.2, \bar{\varepsilon}$ can be considered as serially uncorrelated. Otherwise, a suspicion of a serial correlation is legitimate.

\subsubsection{KS-test, Wald-test and F-test with MATLAB}

In order to perform the KS-test, the kstest MATLAB function is used. The level of significance $\alpha$ is $5 \%$. It is recommended to calculate the p-value in order to make a good interpretation of the result.

To perform the Wald-test, (23) is first calculated and the chi2cdf MATLAB function is used. For instance, with (23), the following instruction is used $p=1-\operatorname{chi} 2 c d f\left(\eta_{\hat{\delta}}^{2}, b\right)$ where $p$ is the $\mathrm{p}$-value. It is checked that $p \geq \alpha$ to validate the set of instruments.

For the F-test, the $f c d f$ MATALAB function is used. $\hat{F}$ given by (24) is first calculated and the following instruction is used $p=1-f c d f(\hat{F}, b-b c, r-b)$ and if $p \geq \alpha$, the model reduction is validated.

\subsection{Brief introduction of the TX40 Robot}

The TX40 robot has a serial structure with six rotational joints and is characterized by a coupling between the joints 5 and 6 . This coupling adds two additional parameters: $f v_{m 6}$ the viscous friction coefficient of motor 6 and $f c_{m 6}$ the dry friction coefficient of motor 6 . The TX40 robot has 60 base dynamic parameters. Its complete modelling is given in (Janot et al. 2014, a).

The robot is PD-controlled and $\tau_{j}$ is given by

$\tau_{j}=g_{\tau_{j}}\left(k_{p_{j}}\left(q_{r_{j}}-q_{\text {mes }_{j}}\right)-k_{v_{j}} \dot{q}_{\text {mes }_{j}}\right)$.

where $k_{p_{j}}$ is the proportional gain in $\mathrm{Nm} / \mathrm{rad}, k_{v_{j}}$ is the derivative gain in $\mathrm{Nm} /(\mathrm{rad} / \mathrm{s}), g_{\tau_{j}}$ is the drive gain and $\dot{q}_{\text {mes }_{j}}$ is the velocity calculated from the differentiation of $q_{m e s_{j}}$. 
The bandwidth of the first (resp. last) three position closed-loops is $10 \mathrm{~Hz}$ (resp. $20 \mathrm{~Hz}$ ). The results obtained with a PID controller sticking to those given in this paper, the use of a PD controller is enough and this is consistent with the results presented in (Gautier et al. 2013).

The reference trajectories $\left(\mathbf{q}_{r}, \dot{\mathbf{q}}_{r}, \ddot{\mathbf{q}}_{r}\right)$ are designed so that $\ddot{\mathbf{q}}_{r}$ are trapezoidal. Since $\operatorname{cond}(\mathbf{X}(\hat{\mathbf{q}}, \hat{\hat{\mathbf{q}}}, \hat{\hat{\mathbf{q}}}))=200,\left(\mathbf{q}_{r}, \dot{\mathbf{q}}_{r}, \ddot{\mathbf{q}}_{r}\right)$ excite well the base parameters (Gautier and Khalil 1992) and (Pressé and Gautier 1993). To evaluate the three identification methods, data are stored with a measurement frequency $f_{m}=5 \mathrm{kHz}$.

To validate the estimates, cross-validations are performed. They are carried out with 3 fifth-order polynomials passing through points different from those defined to build the trajectories used to run the 3 identification methods. For cross-test validations, data are stored with a measurement frequency $f_{m}^{c v}=1 \mathrm{kHz}$ and the relative errors are calculated with the LS or 2SLS estimates and with these trajectories (see (Janot et al. 2014, a) for the details).

\subsection{IDIM-LS method, 2SLS method and regressed DWH-test combined with an appropriate bandpass filtering}

The IDIM-LS method is carried out with a filtered position $\hat{\mathbf{q}}$ calculated with a $40 \mathrm{~Hz}$ fourth-order Butterworth filter. For the three methods, the parallel decimation is carried out with a $10 \mathrm{~Hz}$ Tchebyshef filter.

Before calculating the LS and the 2SLS estimates, the construction of $\hat{\mathbf{Z}}$ is validated with the procedure described in the subsection 3.3. The results are given in Table 1 where $b_{j}$ is the number of identifiable parameters of a joint $j$. Because one has $\eta_{\hat{\delta}}^{2} \leq \chi^{2}(b)$ with a p-value greater than $0.05, \hat{\mathbf{z}}$ is valid and the 2SLS estimates are thus unbiased. For the columns associated with joint accelerations, the $r_{\hat{v}}^{k}$ 's are not null although very small (i.e. less than 1e-3) whereas for the columns associated with joint positions and/or velocities only, the $r_{\dot{v}}^{k}$ 's are null (smaller than 1e-20). A F-test is therefore required to make a final decision.

TABLE 1:

RESULTS OF THE WALD-TEST (23) FOR EACH JOINT J

\begin{tabular}{|c|c|c|c|c|}
\hline Joint $j$ & $b_{j}$ & $\chi^{2}\left(b_{j}\right)$ & $\max \left(\eta_{\hat{\delta}}^{2}\right)$ & $\mathrm{p}$-value \\
\hline 1 & 34 & 48.5 & 18.5 & 0.98 \\
\hline 2 & 37 & 52.3 & 12.4 & 0.99 \\
\hline 3 & 31 & 45.0 & 18.1 & 0.97 \\
\hline 4 & 24 & 36.5 & 5.4 & 0.99 \\
\hline 5 & 20 & 31.3 & 11.7 & 0.93 \\
\hline 6 & 11 & 19.7 & 9.1 & 0.61 \\
\hline
\end{tabular}


The first hypothesis $\overline{\boldsymbol{\varepsilon}} \sim \mathrm{N}\left(\mathbf{0}, \mathbf{I}_{r}\right)$ is validated with the KS-test with a level of significance $\alpha=0.05$. The distribution of $\bar{\varepsilon}$ obtained with the IDIM-LS method and its estimated Gaussian are plotted in Fig. 3 (similar results are obtained with the two others methods). The KS-test accepts $\overline{\boldsymbol{\varepsilon}} \sim \mathrm{N}\left(\mathbf{0}, \mathbf{I}_{r}\right)$ and the distribution of $\overline{\boldsymbol{\varepsilon}}$ matches a Gaussian distribution with the three methods. Furthermore, $d w$ calculated with (25) and given in Table 2 is close to 2.0 with the three methods. $\overline{\boldsymbol{\varepsilon}}$ is thus serially independent with $\overline{\boldsymbol{\varepsilon}} \sim \mathrm{N}\left(\mathbf{0}, \mathbf{I}_{r}\right)$.

The IDIM-LS and the 2SLS estimates are given in Table 2 as well the estimates $\hat{\boldsymbol{\theta}}$ calculated with the augmented DWH-test (NS stands for "Not Significant"). The F-test accepts to cancel the base parameters such that $\% \hat{\sigma}_{\hat{\boldsymbol{\beta}}_{L S}(i)}\left(\right.$ resp. $\left.\% \hat{\sigma}_{\hat{\beta}_{2 S L S}(i)}\right)$ is greater than $30 \%$. Actually, one obtains $\|\bar{\varepsilon}\|=48.5$ with the whole model and $\left\|\bar{\varepsilon}_{c}\right\|=49$ with the reduced model. With $b=60, b_{c}=28$ and $r=2160$, one has $\hat{F} \approx 1.4$ with a $\mathrm{p}$-value greater than 0.05 . From 60 base parameters, only 28 define a set of essential dynamic parameters. Since the F-test accepts $H_{0}: \boldsymbol{\theta}=\mathbf{0}$, relation (15) holds, $\mathbf{X}_{X T D}$ collapses to $\mathbf{X}$ and $\mathbf{X}(\hat{\mathbf{q}}, \hat{\dot{\mathbf{q}}}, \hat{\mathbf{q}}) \approx \mathbf{X}\left(\mathbf{q}_{n f}, \dot{\mathbf{q}}_{n f}, \ddot{\mathbf{q}}_{n f}\right)$. However, the 2SLS estimates are slightly less efficient than the IDIM-LS estimates because one has $\% \hat{\sigma}_{\hat{\beta}_{2 L L}} \geq \% \hat{\sigma}_{\hat{\beta}_{2 L S}}$ for each estimate. This result is consistent with the theory of Statistics (Wooldridge 2009).

Direct comparisons have been performed with the following relative errors: $\%$ rel $l_{\hat{\mathbf{y}}}=\left\|\mathbf{y}-\mathbf{X} \hat{\boldsymbol{\beta}}_{L S}\right\| /\|\mathbf{y}\|$ for the IDIM-LS method, \% rel $_{\hat{y}}=\left\|\mathbf{y}-\mathbf{Z} \hat{\boldsymbol{\beta}}_{\text {LSLS }}\right\| /\|\mathbf{y}\|$ for revised DWH-test and for $\%$ rel $l_{\hat{\mathbf{y}}}=\left\|\mathbf{y}-\mathbf{X}_{X T D} \hat{\boldsymbol{\beta}}_{X T D}\right\| /\|\mathbf{y}\|$ the regressed DWH-test. With relative errors close to $6 \%$ (see Table 2), the matching is therefore good. Cross-test validations have been performed. In Fig. 2, the torque reconstructed with the IDIMLS estimates and with the second trajectory matches the measured one while the norm of the relative error calculated with each validation trajectory and with the IDIM-LS and the 2SLS estimates given in Table 3 stick to those calculated with the direct comparisons. The estimates can be considered as unbiased.

TABLE 2:

IDIM-LS AND 2SLS ESTIMATES, RegRESSEd DWH-TESt EStIMATES - APPROPRIATE DATA FILTERING

\begin{tabular}{|l|l|l|l|}
\hline & $\hat{\boldsymbol{\beta}}_{L S}\left(\% \hat{\sigma}_{\hat{\boldsymbol{\beta}}_{L S}}\right)$ & $\hat{\boldsymbol{\beta}}_{2 S L S}\left(\% \hat{\sigma}_{\hat{\boldsymbol{\beta}}_{2 L L S}}\right)$ & $\hat{\boldsymbol{\theta}}$ \\
\hline $\mathrm{ZZ}_{1 R}$ & $1.26(1.2 \%)$ & $1.25(1.3 \%)$ & $\mathrm{NS}$ \\
\hline $\mathrm{Fv}_{1}$ & $8.1(0.7 \%)$ & $8.20(0.7 \%)$ & $\mathrm{NI}$ \\
\hline $\mathrm{FC}_{1}$ & $6.60(2.3 \%)$ & $6.54(2.6 \%)$ & $\mathrm{NI}$ \\
\hline $\mathrm{XX}_{2 R}$ & $-0.48(2.5 \%)$ & $-0.48(2.9 \%)$ & $\mathrm{NS}$ \\
\hline $\mathrm{XZ}_{2 R}$ & $-0.16(4.4 \%)$ & $-0.16(4.8 \%)$ & $\mathrm{NS}$ \\
\hline $\mathrm{ZZ}_{2 \mathrm{R}}$ & $1.09(1.1 \%)$ & $1.09(1.2 \%)$ & $\mathrm{NS}$ \\
\hline $\mathrm{MX}_{2 \mathrm{R}}$ & $2.20(2.5 \%)$ & $2.21(2.9 \%)$ & $\mathrm{NI}$ \\
\hline $\mathrm{Fv}_{2}$ & $5.68(1.1 \%)$ & $5.68(1.2 \%)$ & $\mathrm{NI}$ \\
\hline $\mathrm{FC}_{2}$ & $7.76(1.8 \%)$ & $7.77(2.1 \%)$ & $\mathrm{NI}$ \\
\hline $\mathrm{XX}_{3 R}$ & $0.13(9.5 \%)$ & $0.13(10.2 \%)$ & $\mathrm{NS}$ \\
\hline $\mathrm{ZZ}_{3 R}$ & $0.12(7.6 \%)$ & $0.12(8.8 \%)$ & $\mathrm{NS}$ \\
\hline $\mathrm{MY}_{3 \mathrm{R}}$ & $-0.59(2.2 \%)$ & $-0.59(2.3 \%)$ & $\mathrm{NI}$ \\
\hline
\end{tabular}




\begin{tabular}{|l|l|l|l|}
\hline $\mathrm{Ia}_{3}$ & $0.084(8.8 \%)$ & $0.088(9.2 \%)$ & $\mathrm{NS}$ \\
\hline $\mathrm{Fv}_{3}$ & $2.02(1.7 \%)$ & $2.03(1.8 \%)$ & $\mathrm{NI}$ \\
\hline $\mathrm{FC}_{3}$ & $6.10(1.8 \%)$ & $6.05(1.9 \%)$ & $\mathrm{NI}$ \\
\hline $\mathrm{MX}_{4}$ & $-0.02(26.7 \%)$ & $-0.02(30.0 \%)$ & $\mathrm{NI}$ \\
\hline $\mathrm{Ia}_{4}$ & $0.029(8.8 \%)$ & $0.029(9.4 \%)$ & $\mathrm{NS}$ \\
\hline $\mathrm{Fv}_{4}$ & $1.14(1.5 \%)$ & $1.15(1.5 \%)$ & $\mathrm{NI}$ \\
\hline $\mathrm{Fc}_{4}$ & $2.34(2.6 \%)$ & $2.27(2.6 \%)$ & $\mathrm{NI}$ \\
\hline $\mathrm{MY}_{5 \mathrm{R}}$ & $-0.03(13.7 \%)$ & $-0.03(14.1 \%)$ & $\mathrm{NI}$ \\
\hline $\mathrm{Ia}_{5}$ & $0.044(8.9 \%)$ & $0.041(11.2 \%)$ & $\mathrm{NS}$ \\
\hline $\mathrm{Fv}_{5}$ & $1.87(1.8 \%)$ & $1.92(2.0 \%)$ & $\mathrm{NI}$ \\
\hline $\mathrm{Fc}_{5}$ & $2.93(3.0 \%)$ & $2.79(3.5 \%)$ & $\mathrm{NI}$ \\
\hline $\mathrm{Ia}_{6}$ & $0.01(9.4 \%)$ & $0.01(10.9 \%)$ & $\mathrm{NS}$ \\
\hline $\mathrm{Fv}_{6}$ & $0.67(1.5 \%)$ & $0.69(1.6 \%)$ & $\mathrm{NI}$ \\
\hline $\mathrm{Fc}_{6}$ & $2.08(2.5 \%)$ & $2.00(2.8 \%)$ & $\mathrm{NI}$ \\
\hline $\mathrm{fv}_{\mathrm{m} 6}$ & $0.63(1.6 \%)$ & $0.63(1.8 \%)$ & $\mathrm{NI}$ \\
\hline $\mathrm{fc}_{\mathrm{m} 6}$ & $1.80(3.7 \%)$ & $1.81(4.2 \%)$ & $\mathrm{NI}$ \\
\hline$\% r e l_{\hat{\mathrm{y}}}$ & $6.0 \%$ & $6.0 \%$ & $6.0 \%$ \\
\hline$d w$ & 1.8 & 1.9 & 1.9 \\
\hline
\end{tabular}

TABLE 3:

RELATIVE ERRORS OBTAINED WITH CROSS-VALIDATION, THE IDIM-LS AND THE 2SLS ESTIMATES

\begin{tabular}{|c|c|c|c|}
\hline & $f_{m}^{c v}$ & $\% \operatorname{rel}_{\hat{\mathrm{y}}}(\mathrm{LS})$ & \% rel $_{\hat{\mathrm{y}}}(2 \mathrm{SLS})$ \\
\hline Trajectory 1 & $1 \mathrm{kHz}$ & $6.5 \%$ & $6.5 \%$ \\
\hline Trajectory 2 & $1 \mathrm{kHz}$ & $6.5 \%$ & $6.5 \%$ \\
\hline Trajectory 3 & $1 \mathrm{kHz}$ & $7.0 \%$ & $7.0 \%$ \\
\hline
\end{tabular}

Cross-validation, joint 1

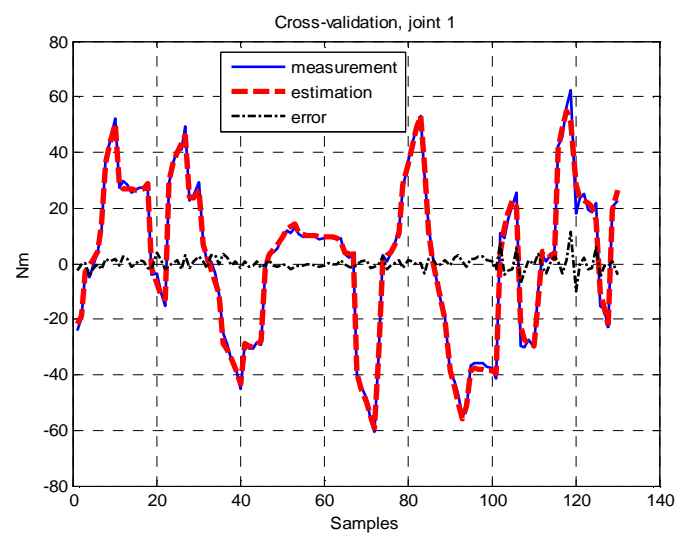

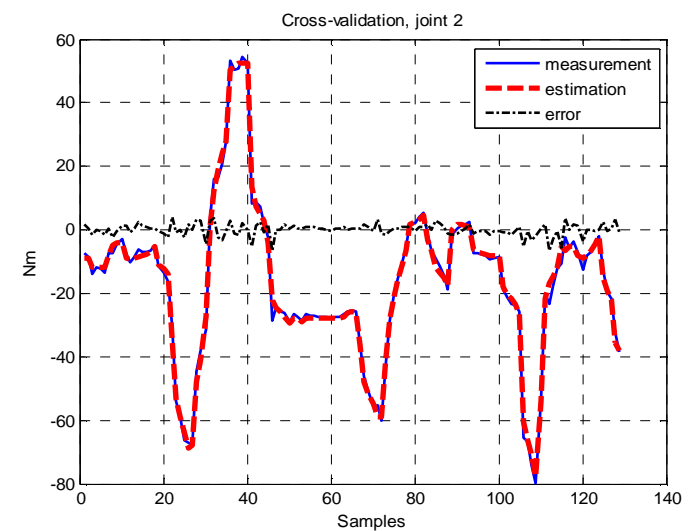



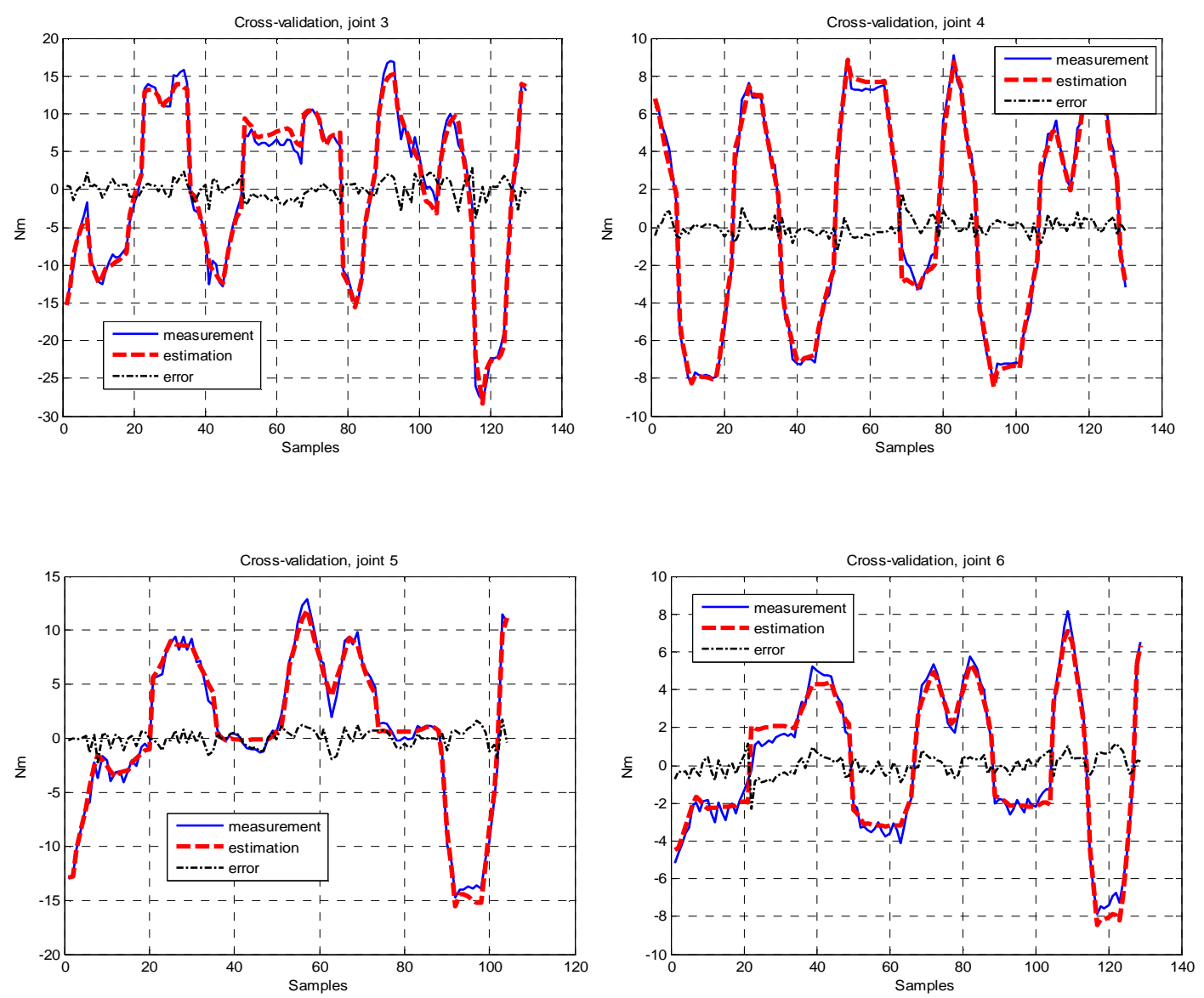

Fig. 2. Cross-validations, joints 1, 2, 3, 4, 5 and 6 with 2SLS estimates and with the first trajectory. Blue: measurement; red: estimation; black: error. Appropriate data filtering. The constructed torques stick to the measured ones. Similar results are obtained with the IDIM-LS method.

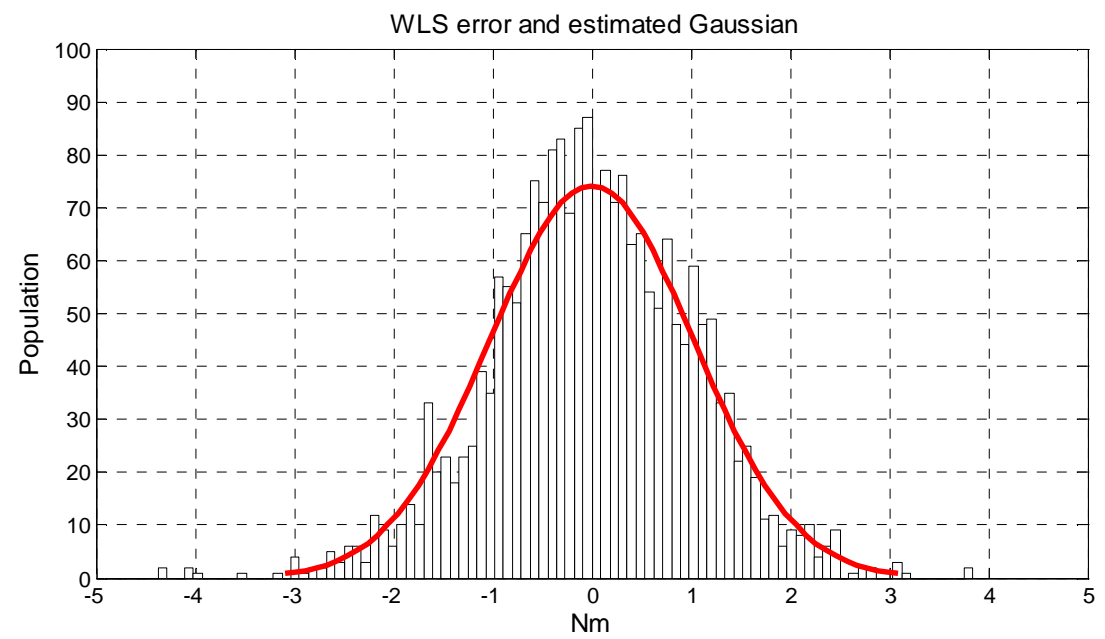

Fig. 3. Histogram of IDIM-LS error and its estimated Gaussian - Appropriate data filtering. The distribution matches a Gaussian distribution. A similar result is obtained with the 2SLS method. 


\subsection{IDIM-LS method, 2SLS method and the regressed DWH-test combined with an inappropriate data filtering}

In this section, the robustness of the methods against an inappropriate data filtering is studied. The IDIM-LS and 2SLS methods are carried out with the position $\hat{\mathbf{q}}$ filtered with a $200 \mathrm{~Hz}$ fourth-order Butterworth filter and with velocities $\hat{\dot{q}}$ and accelerations $\hat{\hat{\mathbf{q}}}$, calculated with a central difference algorithm of $\hat{\mathbf{q}}$. The parallel decimation is carried out with a lowpass Tchebyshef filter with a cutoff frequency of $100 \mathrm{~Hz}$.

Because one has $\eta_{\hat{\delta}}^{2} \leq \chi^{2}(b)$ with a $\mathrm{p}$-value greater than $0.05, \hat{\mathbf{Z}}$ is valid and the 2SLS estimates are thus unbiased. In that case, the $r_{\hat{\mathrm{v}}}^{k}$ 's associated with joint accelerations are of the same magnitude as those of the $r_{\mathrm{x}}^{k}$ 's. With the IDIM-LS method, the 2SLS method and the regressed DWH-test, the KS-test accepts the hypothesis $\overline{\boldsymbol{\varepsilon}} \sim \mathrm{N}\left(\mathbf{0}, \mathbf{I}_{r}\right)$ with a level of significance $\alpha=0.05$ while $d w$ is close to 2.0 (see Table 4). Finally, it comes out that $\overline{\boldsymbol{\varepsilon}}$ is serially independent with $\overline{\boldsymbol{\varepsilon}} \sim \mathrm{N}\left(\mathbf{0}, \mathbf{I}_{r}\right)$.

The estimates of the IDIM-LS, the 2SLS methods and the regressed DWH-test are given in Table 4 (only the significant parameters are given). At first glance, the IDIM-LS estimates seem acceptable because they are not aberrant, the relative error $\%$ rel $_{\hat{y}}$ is not critical and the histogram of IDIM-LS error plotted in Fig. 4 matches a Gaussian distribution. Unfortunately, they are biased since they do not stick to the 2SLS estimates while the observed differences are not spanned by the LS variances and $\boldsymbol{\theta}$ contributes to the dynamics, the F-test rejecting $H_{0}: \boldsymbol{\theta}=\mathbf{0}$. The 2SLS estimates obtained with an inappropriate data filtering are less efficient than those obtained with an appropriate data filtering, their relative deviations being four/five times greater. This result highlights the behaviour of IV estimators: they are able to provide unbiased estimates with very large deviations. This result is consistent with the theory of Statistics (Wooldridge 2009).

All the components of $\hat{\boldsymbol{\theta}}$ corresponding to inertia parameters $\left(Z_{1 R}, X X_{2 R}, X Z_{2 R}, Z Z_{2 R}, X X_{3 R}, Z Z_{3 R}, I_{3}, I_{4}\right.$, $\left.l a_{5}, a_{6}\right)$ and to some gravity parameters $\left(\mathrm{MY}_{3 R}, \mathrm{MX}_{4}, \mathrm{MY}_{5 \mathrm{R}}\right)$ are identifiable and have a significant contribution because the F-test rejects $H_{0}: \boldsymbol{\theta}=\mathbf{0}$. This is due to the fact that their associated columns contain noisy joint accelerations. The augmented DWH-test supports the results of the Revised DWH-test (the estimates of the regressed DWH-test are not given because they stick to $\hat{\boldsymbol{\beta}}_{2 S L S}$ ).

Cross-test validations have been performed and the results obtained with the second trajectory and the IDIM-LS estimates are plotted in Fig. 5. Despite the fact that the errors are not negligible, the reconstruction of torques is quite acceptable and the IDIM-LS estimates are acceptable for a nonexpert in system identification. This result shows that the cross-validations may be not enough to make a final decision. In Table 5, the norms of relative errors calculated with the set of trajectories and with the IDIM-LS (resp. the 2SLS) estimates are given. With the 2SLS estimates, these relative errors match those calculated with the direct comparisons whereas there are some differences with the IDIM-LS estimates although these differences are not as critical as expected. Without running the Revised DWH-test, there are no undisputable evidences to conclude that the IDIM-LS estimates are biased. 


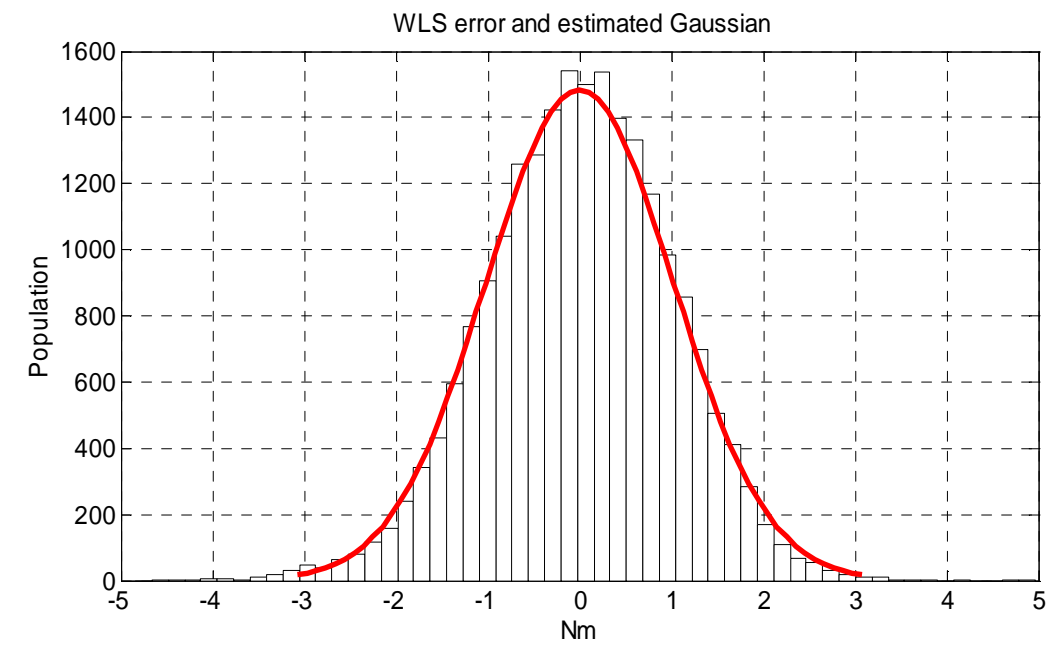

Fig. 4. Histogram of IDIM-LS error with its estimated Gaussian - Inappropriate data filtering. The error distribution matches a Gaussian distribution.

TABLE 4:

IDIM-LS AND 2SLS ESTIMATES, REGRESSED DWH-TEST RESULTS - INAPPROPRIATE DATA FILTERING

\begin{tabular}{|l|l|l|l|}
\hline & $\hat{\boldsymbol{\beta}}_{L S}\left(\% \hat{\sigma}_{\hat{\boldsymbol{\beta}}_{L S}}\right)$ & $\hat{\boldsymbol{\beta}}_{2 S L S}\left(\% \hat{\sigma}_{\hat{\boldsymbol{\beta}}_{2 L L S}}\right)$ & $\hat{\boldsymbol{\theta}}\left(\% \hat{\sigma}_{\hat{\boldsymbol{\theta}}}\right)$ \\
\hline $\mathrm{ZZ}_{1 \mathrm{R}}$ & $1.11(0.8 \%)$ & $1.24(4.1 \%)$ & $-1.22(3 \%)$ \\
\hline $\mathrm{Fv}_{1}$ & $8.23(0.5 \%)$ & $8.25(2.4 \%)$ & $\mathrm{NS}$ \\
\hline $\mathrm{Fc}_{1}$ & $6.42(1.7 \%)$ & $6.38(9.1 \%)$ & $\mathrm{NS}$ \\
\hline $\mathrm{XX}_{2 \mathrm{R}}$ & $-0.38(1.9 \%)$ & $-0.48(10.6 \%)$ & $0.46(9 \%)$ \\
\hline $\mathrm{XZ}_{2 \mathrm{R}}$ & $-0.16(3.0 \%)$ & $-0.16(15.9 \%)$ & $0.14(16 \%)$ \\
\hline $\mathrm{ZZ}_{2 \mathrm{R}}$ & $0.88(0.8 \%)$ & $1.08(3.8 \%)$ & $-1.0(3 \%)$ \\
\hline $\mathrm{MX}_{2 \mathrm{R}}$ & $2.42(1.7 \%)$ & $2.22(9.9 \%)$ & $\mathrm{NS}$ \\
\hline $\mathrm{Fv}_{2}$ & $5.63(0.8 \%)$ & $5.75(4.4 \%)$ & $\mathrm{NS}$ \\
\hline $\mathrm{Fc}_{2}$ & $7.88(1.3 \%)$ & $7.55(6.4 \%)$ & $\mathrm{NS}$ \\
\hline $\mathrm{XX_{3R }}$ & $0.19(5.7 \%)$ & $0.13(29.3 \%)$ & $-0.11(20 \%)$ \\
\hline $\mathrm{ZZ}_{3 \mathrm{R}}$ & $0.07(6.2 \%)$ & $0.11(28.8 \%)$ & $-0.12(10 \%)$ \\
\hline $\mathrm{MY}_{3 R}$ & $-0.71(1.0 \%)$ & $-0.60(6.6 \%)$ & $0.5(6 \%)$ \\
\hline $\mathrm{Ia}_{3}$ & $0.15(2.6 \%)$ & $0.09(24.5 \%)$ & $-0.07(20 \%)$ \\
\hline $\mathrm{Fv}_{3}$ & $2.03(1.0 \%)$ & $2.01(4.5 \%)$ & $\mathrm{NS}$ \\
\hline $\mathrm{Fc}_{3}$ & $5.96(1.1 \%)$ & $5.83(5.1 \%)$ & $\mathrm{NS}$ \\
\hline $\mathrm{MX}_{4}$ & $-0.01(20.1 \%)$ & $-0.02(27.5 \%)$ & $0.01(50 \%)$ \\
\hline $\mathrm{Ia}_{4}$ & $0.022(3.9 \%)$ & $0.028(25.5 \%)$ & $\mathrm{NS}$ \\
\hline $\mathrm{Fv}_{4}$ & $1.14(0.6 \%)$ & $1.17(3.2 \%)$ & $\mathrm{NS}$ \\
\hline $\mathrm{Fc}_{4}$ & $2.35(1.0 \%)$ & $2.23(6.3 \%)$ & $\mathrm{NS}$ \\
\hline $\mathrm{MY}_{5 \mathrm{R}}$ & $-0.02(5.7 \%)$ & $-0.03(28.3 \%)$ & $0.03(9 \%)$ \\
\hline $\mathrm{Ia}_{5}$ & $0.02(3.2 \%)$ & $0.04(25.2 \%)$ & $-0.03(12 \%)$ \\
\hline $\mathrm{Fv}_{5}$ & $1.84(0.7 \%)$ & $1.94(4.0 \%)$ & $\mathrm{NS}$ \\
\hline $\mathrm{Fc}_{5}$ & $3.01(1.1 \%)$ & $2.72(7.3 \%)$ & $\mathrm{NS}$ \\
\hline $\mathrm{Ia}_{6}$ & $0.007(3.3 \%)$ & $0.01(24.5 \%)$ & $-0.008(10 \%)$ \\
\hline $\mathrm{Fv}_{6}$ & $0.67(0.6 \%)$ & $0.69(3.8 \%)$ & $\mathrm{NS}$ \\
\hline $\mathrm{Fc}_{6}$ & $2.11(1.0 \%)$ & $1.97(6.2 \%)$ & $\mathrm{NS}$ \\
\hline $\mathrm{fv}_{\mathrm{m} 6}$ & $0.63(0.6 \%)$ & $0.64(3.8 \%)$ & $\mathrm{NS}$ \\
\hline $\mathrm{fc}_{\mathrm{m} 6}$ & $1.80(1.4 \%)$ & $1.74(8.1 \%)$ & $\mathrm{NS}$ \\
\hline & & & \\
\hline
\end{tabular}




\begin{tabular}{|l|l|l|l|}
\hline \%rel $_{\mathrm{y}}$ & $17.0 \%$ & $12.5 \%$ & $11.0 \%$ \\
\hline$d w$ & 1.7 & 1.8 & 1.8 \\
\hline
\end{tabular}

TABLE 5:

RELATIVE ERRORS OBTAINED WITH CROSSCHECKING, IDIM-LS AND 2SLS ESTIMATES

\begin{tabular}{|l|c|c|c|}
\hline & $f_{m}^{c v}$ & rrel $_{\hat{\mathrm{y}}}(\mathrm{LS})$ & ${\text { \% } \text { rel }_{\hat{\mathrm{y}}}}(2 \mathrm{SLS})$ \\
\hline Trajectory 1 & $1 \mathrm{kHz}$ & $20.0 \%$ & $14.0 \%$ \\
\hline Trajectory 2 & $1 \mathrm{kHz}$ & $22.0 \%$ & $14.0 \%$ \\
\hline Trajectory 3 & $1 \mathrm{kHz}$ & $21.0 \%$ & $14.5 \%$ \\
\hline
\end{tabular}
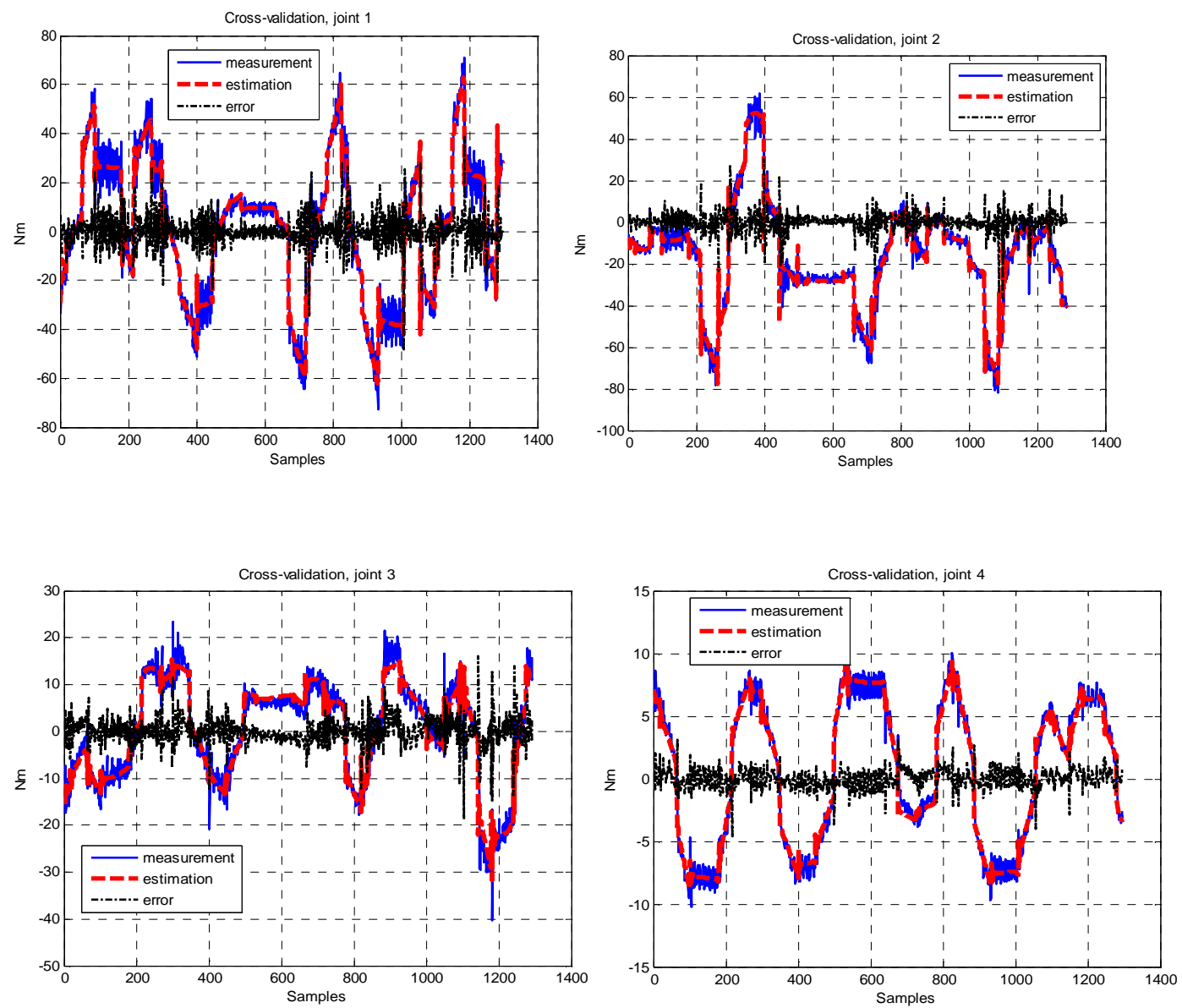

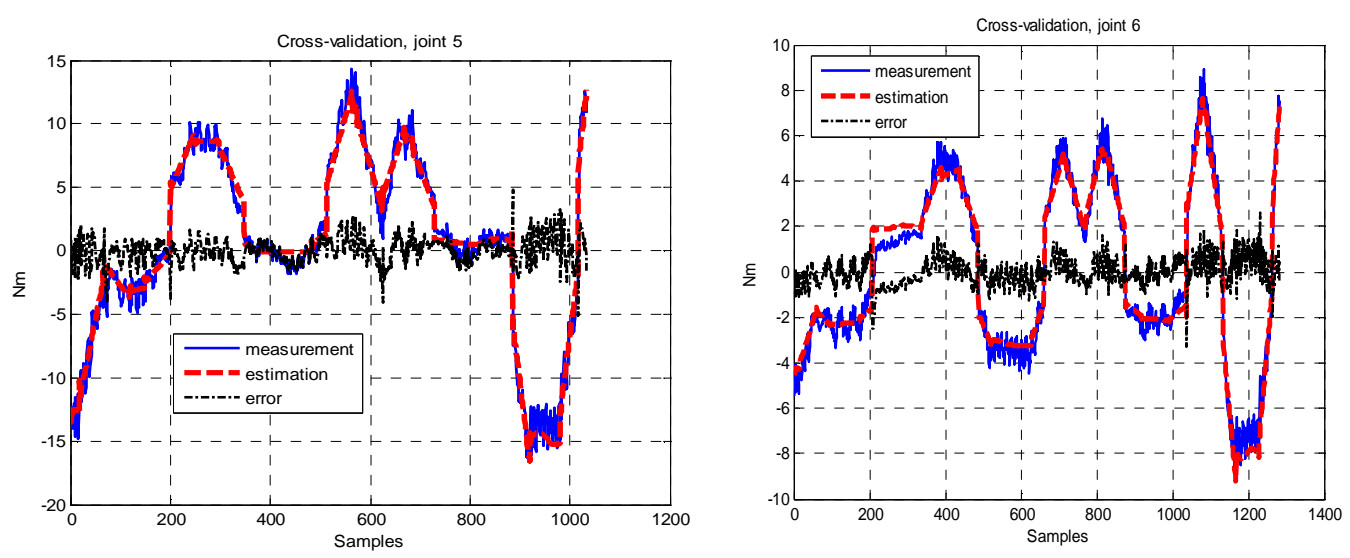

Fig. 5. Cross-validations, joints 1, 2, 3, 4, 5 and 6 with IDIM-LS estimates and with the second trajectory. Blue: measurement; red: estimation; black: error. Inappropriate data filtering. The matching is quite good despite the fact that the IDIM-LS estimates are biased.

\subsection{Robustness against a misspecified model}

The robustness of the Revised DWH-test against a misspecified model is now studied. Because the gear ratios are greater than 25 , it is legitimate to assume that the parameters of gravity and the offdiagonal elements of inertia matrices do not contribute significantly to the dynamics. These parameters and their associated columns are removed from the IDM. The data are filtered as explained in Section 4.3 .

For the inertia parameters of joints 1, 2, 3 and 4, the Wald-test rejects the hypothesis that $\hat{\mathbf{z}}$ is valid because the minimum of $\eta_{\hat{\delta}}^{2}$ given in Table 6 is greater than $\chi^{2}\left(b_{j}\right)$ while the $\mathrm{p}$-value is almost null. Interestingly, the set of instruments of joint 5 and 6 is valid. This is mainly due to the fact that the gravity parameters and the off-diagonal elements of inertia matrices are practically null. Because $\hat{\mathbf{Z}}$ is not valid, the 2SLS estimates are biased.

The IDIM-LS and 2SLS estimates given in Table 7 differ from those given in Table 2. They are therefore biased. The KS-test rejects the hypothesis $\overline{\boldsymbol{\varepsilon}} \sim \mathrm{N}\left(\mathbf{0}, \mathbf{I}_{r}\right)$ for both methods. The IDIM-LS error and its estimated Gaussian are plotted in Fig. 6 and the distribution does not match a Gaussian distribution (a similar result is obtained with the 2SLS method). This experiment shows that the Revised DWH-test is able to detect a model misspecification.

TABLE 6:

RESULTS OF THE WALD-TEST (23) FOR THE JOINTS 1, 2, 3 AND 4 - MISSPECIFIED MODEL - APPROPRIATE DATA FILTERING

\begin{tabular}{|l|l|l|l|l|}
\hline Joint $j$ & $b_{j}$ & $\chi^{2}\left(b_{j}\right)$ & $\min \left(\eta_{\hat{\delta}}^{2}\right)$ & p-value \\
\hline
\end{tabular}




\begin{tabular}{|c|c|c|c|c|}
\hline 1 & 3 & 7.81 & 16.3 & $\sim 0$ \\
\hline 2 & 3 & 7.81 & 19.1 & $\sim 0$ \\
\hline 3 & 4 & 9.5 & 25.7 & $\sim 0$ \\
\hline 4 & 4 & 9.5 & 19.6 & $\sim 0$ \\
\hline 5 & 4 & 9.5 & 5.1 & 0.28 \\
\hline 6 & 6 & 12.59 & 4.9 & 0.56 \\
\hline
\end{tabular}

TABLE 7:

IDIM-LS ESTIMATES AND 2SLS ESTIMATES - MISSPECIFIED MODEL AND APPROPRIATE DATA FILTERING

\begin{tabular}{|l|l|l|}
\hline & $\hat{\boldsymbol{\beta}}_{L S}\left(\% \hat{\sigma}_{\hat{\boldsymbol{\beta}}_{L S}}\right)$ & $\hat{\boldsymbol{\beta}}_{2 S L S}\left(\% \hat{\sigma}_{\hat{\boldsymbol{\beta}}_{\text {SLS }}}\right)$ \\
\hline $\mathrm{ZZ}_{1 \mathrm{R}}$ & $1.10(3.0 \%)$ & $1.08(3.5 \%)$ \\
\hline $\mathrm{Fv}_{1}$ & $8.16(3.0 \%)$ & $8.17(3.6 \%)$ \\
\hline $\mathrm{Fc}_{1}$ & $6.50(10.6 \%)$ & $6.48(11.0 \%)$ \\
\hline $\mathrm{ZZ}_{2 \mathrm{R}}$ & $1.37(2.3 \%)$ & $1.20(2.0 \%)$ \\
\hline $\mathrm{Fv}_{2}$ & $5.80(5.2 \%)$ & $5.83(5.8 \%)$ \\
\hline $\mathrm{Fc}_{2}$ & $6.80(10.3 \%)$ & $6.80(11.0 \%)$ \\
\hline $\mathrm{ZZ}_{3 \mathrm{R}}$ & $0.31(7.8 \%)$ & $0.27(6.7 \%)$ \\
\hline $\mathrm{Ia}_{3}$ & $0.05(36.0 \%)$ & $0.07(40.0 \%)$ \\
\hline $\mathrm{Fv}_{3}$ & $2.21(7.2 \%)$ & $2.22(7.6 \%)$ \\
\hline $\mathrm{Fc}_{3}$ & $5.55(9.3 \%)$ & $5.53(9.5 \%)$ \\
\hline $\mathrm{Ia}_{4}$ & $0.04(26.2 \%)$ & $0.05(31.1 \%)$ \\
\hline $\mathrm{Fv}_{4}$ & $1.18(5.0 \%)$ & $1.20(5.8 \%)$ \\
\hline $\mathrm{Fc}_{4}$ & $2.20(9.6 \%)$ & $2.17(10.0 \%)$ \\
\hline $\mathrm{Ia}_{5}$ & $0.06(28.2 \%)$ & $0.05(29.3 \%)$ \\
\hline $\mathrm{Fv}_{5}$ & $1.90(7.1 \%)$ & $1.89(7.3 \%)$ \\
\hline $\mathrm{Fc}_{5}$ & $2.75(12.5 \%)$ & $2.75(12.6 \%)$ \\
\hline $\mathrm{Ia}_{6}$ & $0.01(31.0 \%)$ & $0.01(33.0 \%)$ \\
\hline $\mathrm{Fv}_{6}$ & $0.69(5.1 \%)$ & $0.69(5.4 \%)$ \\
\hline $\mathrm{Fc}_{6}$ & $2.0(8.9 \%)$ & $2.0(9.3 \%)$ \\
\hline $\mathrm{fv}_{\mathrm{m} 6}$ & $0.64(5.6 \%)$ & $0.64(5.9 \%)$ \\
\hline $\mathrm{fc}_{\mathrm{m} 6}$ & $1.70(15.2 \%)$ & $1.70(16.0 \%)$ \\
\hline$\% r e l_{\hat{\mathbf{y}}}$ & $17.0 \%$ & $21.0 \%$ \\
\hline$d w$ & 1.8 & 1.8 \\
\hline & & \\
\hline
\end{tabular}




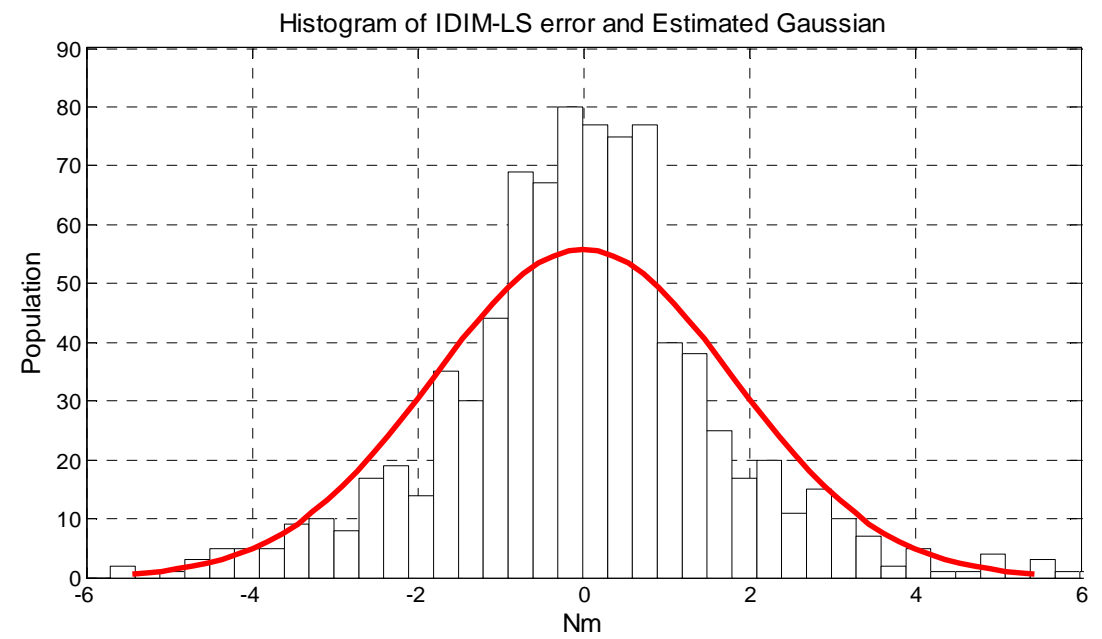

Fig. 6. Histogram of IDIM-LS error and its estimated Gaussian - Appropriate data filtering Misspecified dynamic model

\section{Conclusion}

In this paper, a Revised DWH-test suitable for identification of robots was introduced and experimentally validated on a 6 degrees-of-freedom industrial robot. The main contributions of the work presented in this paper are the following:

- The Revised DWH-test can validate/invalidate the instruments chosen by the user and is based on general statistical assumptions,

- The Revised is able to detect model misspecifications,

- The algorithm makes use of the QR factorization of an augmented matrix and is combined with a F-test if required,

- The Revised DWH-test is able to validate/invalidate IDIM-LS estimates.

The results provided by the revised statistic were cross-validated and compared with those provided by the augmented DWH-test widely used in Econometrics. Since all the results are close to each others, this shows that the results provided by the Revised DWH-test are reliable.

Future works will address the application of the Revised DWH-test on flexible robots and electrical motors. The calculation of the optimal prefilters for robot identification and the application of the experiment design are worth of investigation and will be addressed.

\section{References}

Aguero and Goodwin (2006), "On the Optimality of Open and Closed Loop Experiments in System Identification," In: Proceedings of the 45th IEEE Conference on Decision and Control Conference, 2006, Page(s): $163-168$

Aguero and Goodwin (2007), "Choosing between open- and closed-loop experiments in linear system identification," IEEE Transactions on Automatic Control, Vol. 52(8), August 2007, pp. 1475-14780 
Calanca A., Capisani L. M., Ferrara A., and Magnani L. (2011), "MIMO Closed Loop Identification of an Industrial Robot," IEEE Transactions on Control System Technology, Vol. 19(5), September 2011, pp. 1214-1224.

Chen F., Garnier, H. and Gilson, M., Robust identification of continuous-time models with arbitrary time-delay from irregularly sampled data (2015), Journal of Process Control, vol. 25, pp. 19-27, January 2015.

Davidson R. and MacKinnon J.G. (1993), "Estimation and Inference in Econometrics," Oxford University Press, New York, 1993.

Dellon B. and Matsuoka Y. (2009), "Modeling and System Identification of a Life-Size Brake-Actuated Manipulator," IEEE Transactions on Robotics, vol. 25(3), June 2009, pp. 481 - 491.

Garnier H. and Wang L. (2008), "Identification of Continuous-time Models from Sampled Data," Springer, 2008.

Gautier M. (1991), "Numerical calculation of the base inertial parameters," Journal of Robotics Systems, vol. 8, no. 4, pp. 485-506, 1991.

Gautier M. and Khalil W. (1992), "Exciting trajectories for the identification of the inertial parameters of robots," International Journal of Robotics Research, vol. 11, Aug. 1992, pp. 362-375.

Gautier M. and Poignet P. (2001), "Extended Kalman Filtering and Weighted Least-squares Dynamic Identification of Robot," Control Engineering Practice, vol. 9, 2001, pp. 1361-1372.

Gautier M., Janot A., and Vandanjon P.O., (2013), “A New Closed-Loop Output Error Method for Parameter Identification of Robot Dynamics," IEEE Transactions on Control System Technology, vol. 21(2), March 2013, pp. 428-444.

Gilson M., Garnier H., Young P.C. and Van den Hof P. (2011), "Optimal instrumental variable method for closed-loop identification”, Control Theory \& Applications, IET, vol. 5(10), pp. 1147 - 1154.

Hausman J.A. (1978), "Specification Tests in Econometrics," Econometrica, vol. 46(6), 1978, pp. 1251 $-1271$.

Hollerbach J., Khalil W., and Gautier M. (2008), "Model Identification," Springer Handbook of Robotics, Springer, 2008.

Indri M., Calafiore G., Legnani G., Jatta F. and Visioli A. (2002), "Optimized Dynamic Calibration of a SCARA Robot," In: Proceedings of the 15th IFAC World Congress, July 2002, Barcelona, Spain.

Janot A., Vandanjon P.O. and Gautier M. (2013), "A Durbin-Wu-Hausman Test for Industrial Robots Identification," In: Proc. of IEEE International Conference on Robotics and Automation, Karlsruhe, Germany, May 2013, pp. 2956-2961.

Janot A., Vandanjon P.O. and Gautier M. (2014, a), "A Generic Instrumental Variable Approach for Industrial Robots Identification," IEEE Transactions on Control Systems Technology, Vol. 22(1), pp.132-145, January 2014. 
Janot A., Vandanjon P.O. and Gautier M. (2014, b), "An instrumental variable approach for rigid industrial robots identification," Control Engineering Practice, Vol. 25, pp.85-101, April 2014.

Khalil W. and Dombre E. (2002), "Modeling, identification and control of robots", Hermes Penton, London, 2002

Kostic D., de Jager B., Steinbuch M., and Hensen R. (2004), "Modeling and Identification for HighPerformance Robot Control: An RRR-Robotic Arm Case Study," IEEE Transactions on Control System Technology, Vol. 12(6), November 2004.

Landau I. D. and Karimi A. (1997), "An output error recursive algorithm for unbiased identification in closed loop," Automatica, vol. 33, pp. 933-938, 1997.

Landau I. D. (2001), "Identification in closed loop: a powerful design tool (better design models, simpler controllers)," Control Engineering Practice, vol. 9, pp. 51-65, 2001.

Mayeda H., Yoshida K., and Osuka K. (1990), "Base parameters of manipulator dynamic models," IEEE Transactions on Robotics and Automation, vol. 6(3), 1990, pp. 312-321.

Olsen M. M., Swevers J. and Verdonck W. (2002), "Maximum Likelihood Identification of a Dynamic Robot Model: Implementation Issues," International Journal of Robotics Research, vol. 21(2), Feb. 2002, pp. $89-96$.

Östring M., Gunnarsson S., and Norrlöf M. (2003), "Closed-loop identification of an industrial robot containing flexibilities," Control Engineering Practice, vol. 11, pp. 291-300, 2003.

Pressé C. and Gautier M. (1993), "New criteria of exciting trajectories for robot identification," in Proc. 1993 IEEE International Conference on Robotics and Automation, Atlanta, GA, USA, 1993, pp. 907-912

Ramdani N. and Poignet P. (2005), "Robust Dynamic Experimental Identification of Robots with Set Membership Uncertainty," IEEE/ASME Transactions on Mechatronics, vol. 10(2), April 2005, pp. 253 256.

Sargan J.D. (1958), "The Estimation of Economic Relationships Using Instrumental Variables," Econometrica, vol. 26, 1958, pp. 393-415.

Söderström T. and Stoica P. (1989), "System Identification," Prentice Hall international series in systems and control engineering, Great Britain, 1989.

Soewandito D. B., Oetomo D., Ang Jr M.H. (2011), "Neuro-adaptive motion control with velocity observer in operational space formulation," Robotics and Computer-Integrated Manufacturing, Vol. 27(4), August 2011, pp. 829-842.

Swevers J., Ganseman C., Tükel D.B., De Schutter J. and Van Brussel H. (1997), "Optimal Robot Excitation and Identification," IEEE Transactions on Robotics, vol. 13(5), October 1997, pp. 730-740.

Swevers J., Verdonck W., and De Schutter J. (2007), "Dynamic model identification for industrial robots - Integrated experiment design and parameter estimation," IEEE Control Systems Magazine, vol. 27, 2007, pp. 58-71. 
Ting J-A., Mistry M., Peters J., Schaal S. and Nakanishi J. (2006), "A Bayesian Approach to Nonlinear Parameter Identification for Rigid Body Dynamics," in Proceedings of Robotics: Science and Systems, 2006.

Wernholt E. and Gunnarsson S. (2008), "Estimation of Nonlinear Effects in Frequency Domain Identification of Industrial Robots," Instrumentation and Measurement, IEEE Transactions on, vol. 57, pp. 856-863, 2008.

White H. (1980), "A heteroskedasticity-consistent covariance matrix estimator and a direct test for heteroskedasticity," Econometrica, vol. 48(4), pp. 817-838.

Wooldridge J. M. (2009), "Introductory Econometrics: A Modern Approach," Cengage Learning Inc., 4th Edition, 2009.

Xi, F. (1995), "Effect of Non-Geometric Errors on Manipulator Inertial Calibration," In Proc. of International Conference on Robotics and Automation, May 1995, Nagoya, Japan, pp. 1808 - 1813.

Young P.C. (2011), "Recursive Estimation and Time-Series Analysis: An Introduction for the Student and Practitioner," Springer Verlag, 2nd Edition., 2011. 